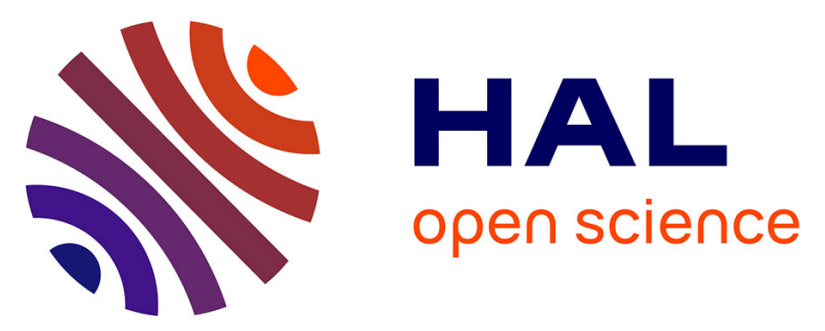

\title{
Velocity-based cardiac contractility personalization from images using derivative-free optimization
}

Ken C.L. Wong, Maxime Sermesant, Kawal Rhode, Matthew Ginks, C. Aldo Rinaldi, Reza Razavi, Hervé Delingette, Nicholas Ayache

\section{- To cite this version:}

Ken C.L. Wong, Maxime Sermesant, Kawal Rhode, Matthew Ginks, C. Aldo Rinaldi, et al.. Velocitybased cardiac contractility personalization from images using derivative-free optimization. Journal of the mechanical behavior of biomedical materials, 2015, 43, pp.35-52. 10.1016/j.jmbbm.2014.12.002 . hal-01095725

\section{HAL Id: hal-01095725 \\ https://inria.hal.science/hal-01095725}

Submitted on 16 Dec 2014

HAL is a multi-disciplinary open access archive for the deposit and dissemination of scientific research documents, whether they are published or not. The documents may come from teaching and research institutions in France or abroad, or from public or private research centers.
L'archive ouverte pluridisciplinaire HAL, est destinée au dépôt et à la diffusion de documents scientifiques de niveau recherche, publiés ou non, émanant des établissements d'enseignement et de recherche français ou étrangers, des laboratoires publics ou privés. 


\title{
Velocity-Based Cardiac Contractility Personalization from Images using Derivative-Free Optimization
}

\author{
Ken C. L. Wonga ${ }^{\mathrm{a}}$, Maxime Sermesant ${ }^{\mathrm{a}, *}, \mathrm{Kawal} \mathrm{Rhode}^{\mathrm{b}}$, Matthew Ginks ${ }^{\mathrm{b}}$, C. Aldo Rinaldi ${ }^{\mathrm{c}}$, Reza Razavi ${ }^{\mathrm{b}}$, Hervé Delingette ${ }^{\mathrm{a}}$, \\ Nicholas Ayache ${ }^{\mathrm{a}}$
}

${ }^{a}$ INRIA, Asclepios Project, 2004 route des Lucioles, 06902 Sophia Antipolis, France

${ }^{b}$ King's College London, Division of Imaging Sciences, St. Thomas' Hospital, London, UK

${ }^{c}$ Department of Cardiology, St. Thomas' Hospital, London, UK

\begin{abstract}
Model personalization is a key aspect for biophysical models to impact clinical practice, and cardiac contractility personalization from medical images is a major step in this direction. Existing gradient-based optimization approaches show promising results of identifying the maximum contractility from images, but the contraction and relaxation rates are not accounted for. A main reason is the limited choices of objective functions when their gradients are required. For complicated cardiac models, analytical evaluations of gradients are very difficult if not impossible, and finite difference approximations are computationally expensive and may introduce numerical difficulties. By removing such limitations with derivative-free optimization, we found that a velocity-based objective function can properly identify regional maximum contraction stresses, contraction rates, and relaxation rates simultaneously with intact model complexity. Experiments on synthetic data show that the parameters are better identified using the velocity-based objective function than its position-based counterpart, and the proposed framework is insensitive to initial parameters with the adopted derivative-free optimization algorithm. Experiments on clinical data show that the framework can provide personalized contractility parameters which are consistent with the underlying physiologies of the patients and healthy volunteers.
\end{abstract}

Keywords: Cardiac contractility, cardiac electromechanical model, derivative-free optimization, model personalization, parameter estimation.

\section{Introduction}

Cardiac model personalization is a process to obtain a biophysical model which accounts for the subject-specific cardiac physiology, and is usually realized as parameter estimation. Given a generic cardiac model derived from invasive experiments of anatomy, electrophysiology, and cardiac mechanics, the model parameters are estimated from subject-specific in vivo measurements such as non-contact endocardial maps and magnetic resonance images (MRI). As simulation of the whole organ has reached a degree of realism which is quantitatively comparable with available cardiac images and signals acquired routinely on patients, model personalization gives a potential impact to clinical practice by improving disease diagnosis and therapy planning, such as cardiac resynchronization therapy (Sermesant et al., 2012).

Cardiac mechanics is the interaction among active contraction stresses, passive mechanical properties (stiffness), and boundary conditions exerted by surrounding anatomical structures (Germann and Stanfield, 2005; Glass et al., 1991). Various cardiac electromechanical models have been proposed to describe such

*Corresponding author at: INRIA, Asclepios Project, 2004 route des Lucioles, 06902 Sophia Antipolis, France. Tel.: +3349238 78 11. Fax: +33492 387669 .

Email addresses: kenclwong@gmail . com (Ken C. L. Wong), maxime.sermesanteinria.fr (Maxime Sermesant) an interaction with different physiological plausibilities, complexities, and computational efficiencies (Nash, 1998; Sermesant et al., 2006a; Usyk et al., 2002; Wong et al., 2010b). According to the characteristics of the models, different personalization algorithms have been proposed. In $\mathrm{Hu}$ et al. (2003), the homogeneous and transversely isotropic Young's moduli of a piece-wise linear passive mechanical model were estimated with the active contraction using an expectation-maximization (EM) algorithm from tagged MRI. In Liu and Shi (2009), the heterogeneous and isotropic Young's moduli and Poisson's ratios were estimated simultaneously with cardiac deformation using an extended Kalman filter-based EM algorithm from cine and tagged MRI. In Chabiniok et al. (2011), the regional maximum contraction stresses were estimated using reduced-order unscented Kalman filtering (rUKF) from porcine cine MRI. In Wang et al. (2009), homogeneous passive material stiffness of a nonlinear transversely isotropic model was estimated with a sequential quadratic programming (SQP) method from tagged MRI and ex vivo DT-MRI of canine hearts. In Xi et al. (2011), the performances of rUKF and SQP for the estimation of stiffness parameters were compared on synthetic data. In Marchesseau et al. (2013), a method based on the unscented transform algorithm was proposed to calibrate the global mechanical parameters of the Bestel-Clément-Sorine electromechanical model (Bestel et al., 2001), followed by the personalization of the regional maximum contraction stresses using rUKF. 
In this paper, we concentrate on cardiac contractility personalization. While efforts have been spent on estimating maximum contraction stresses ( $\mathrm{Hu}$ et al., 2003; Chabiniok et al., 2011; Delingette et al., 2012), the estimations of contraction and relaxation rates have seldom been studied. As these rates are related to clinically important measurements such as the rates of blood pressure (Rushmer et al., 1964; Mason et al., 1971), they are important parameters which should also be estimated. Nevertheless, this is not a trivial task. For example, in our previous work (Delingette et al., 2012), the gradient-based quasi-Newton L-BFGS-B algorithm was utilized to optimize a position-based objective function. Although the utilized adjoint method allows efficient computation of the gradient, it requires the system derivatives of the complicated cardiac electromechanical model. This limits the exploration of the proper objective functions and also the types of parameters to be estimated, as some objective functions are highly nonlinear with respect to the desired parameters. Therefore, only the maximum contraction stresses were estimated in Delingette et al. (2012) even after some model simplifications.

In consequence, we propose the use of derivative-free optimization for cardiac contractility personalization. Without the analytical, numerical, and computational difficulties associated with gradient evaluation, objective functions which may provide better parameter estimation can be investigated with relative ease. By using derivative-free optimization, we propose a velocity-based objective function for simultaneous estimation of regional maximum contraction stresses, rates of contraction, and rates of relaxation. Experiments were performed on synthetic data to show the capability of the framework in identifying regional contractility, and its sensitivity to noise and initial parameters. Experiments on patient and volunteer data also show its clinical relevance.

\section{Cardiac Electromechanical Model}

In the computational environment, the personalized cardiac geometry can be represented as points bounded by surfaces. Using numerical methods such as finite element methods (FEM) (Bathe, 1996; Sermesant et al., 2006a) or meshfree methods (Belytschko et al., 1996; Wong et al., 2010b), the dynamics of a cardiac electromechanical model can be given as:

$$
\mathbf{M U ̈}+\mathbf{C} \dot{\mathbf{U}}+\mathbf{K U}=\mathbf{F}_{b}+\mathbf{F}_{c}
$$

where $\mathbf{M}, \mathbf{C}$, and $\mathbf{K}$ are the mass, damping, and stiffness matrices, and $\ddot{\mathbf{U}}, \dot{\mathbf{U}}$, and $\mathbf{U}$ are the acceleration, velocity, and displacement vectors. $\mathbf{F}_{b}$ comprises the displacement and pressure boundary conditions. $\mathbf{F}_{c}$ is the active contraction force vector derived from electrophysiology and tissue structure. The electromechanical model in Sermesant et al. (2006a) is used in this paper. To facilitate the complicated inverse problem, the stiffness matrix $\mathbf{K}$ describes linear elasticity, though tissue anisotropy is considered. Therefore, the passive mechanical properties are characterized through the Young's moduli $\left(E_{f}\right.$, $\left.E_{c f}\right)$ and Poisson's ratios $\left(\nu_{f}, \nu_{c f}\right)$ along and across the fibers.

The force vector $\mathbf{F}_{b}$ comprises the displacement and pressure boundary conditions (Sermesant et al., 2006a). To hold the mesh in place, displacement boundary conditions are applied to the FEM nodes at the fibrous structure around the valves, and also to some FEM nodes at the apex. These boundary conditions are applied through the penalty method (Bathe, 1996), which is equivalent to attaching a FEM node to one end of a spring which has adjustable stiffness (penalty), with the other end of the spring fixed at the original nodal position. To simulate the four cardiac phases, including filling, isovolumetric contraction, ejection, and isovolumetric relaxation, pressures and penalty constraints are applied to the endocardia of the left and right ventricles. For filling, pressures equal to the mean pressures of the atria are applied. For isovolumetric contraction and relaxation, penalty constraints are applied to keep the ventricular volumes constant. For ejection, pressures equal to the mean pressures of the aorta and the pulmonary artery, which are computed using the three-element Windkessel model with the blood volume changes, are applied. The ventricular pressures of the cardiac cycle can be obtained by combining the ventricular pressures of all phases. As the isovolumetric constraints and the Windkessel model depend on the cardiac kinematics and mechanics, different active contraction forces produce different ventricular pressures.

To obtain $\mathbf{F}_{c}$, the relation between the action potential and the active contraction can be modeled as (Sermesant et al., 2006a):

$$
\frac{\partial \sigma_{c}}{\partial t}+\sigma_{c}=u \sigma_{0}
$$

with $\sigma_{c}$ the contraction stress, $u$ the normalized action potential, and $\sigma_{0}$ the maximum contraction parameter defining the maximum value of $\sigma_{c}$. As $u$ is between 0 and 1 and the changes of depolarization and repolarization are abrupt, the solution of (2) can be approximated as:

$$
\begin{cases}\sigma_{c}(t)=\sigma_{0}\left(1-e^{\alpha_{c}\left(T_{d}-t\right)}\right) & \text { if } T_{d} \leq t \leq T_{r} \\ \sigma_{c}(t)=\sigma_{c}\left(T_{r}\right) e^{\alpha_{r}\left(T_{r}-t\right)} & \text { if } T_{r}<t<T_{d}+H P\end{cases}
$$

where $H P$ is the heart period. $\alpha_{c}$ and $\alpha_{r}$ are the contraction and relaxation rates added to better control the change of $\sigma_{c}$. $T_{d}$ and $T_{r}$ are the depolarization and repolarization times derived from the action potential, and a time constant can be added to model the delay between the electrical and mechanical phenomena. In this paper, the parameters of interest are $\sigma_{0}, \alpha_{c}$, and $\alpha_{r}$. Although this model is relatively simple, it is capable of simulating realistic cardiac cycles (Sermesant et al., 2006a), and its simplicity facilitates the complicated parameter estimation. With $\mathbf{F}_{c}$ providing the active contraction, $\mathbf{F}_{b}$ providing the boundary conditions, and $\mathbf{M}, \mathbf{C}, \mathbf{K}$ providing the passive mechanical properties, the cardiac cycle can be simulated.

\section{Electrophysiology and Kinematics Personalization}

\subsection{Electrophysiology Personalization}

To avoid accumulating sources of uncertainties, patient-specific data sets including a rich description of cardiac electrophysiology were utilized. In addition to the acquisition of anatomical and cine MRI, non-contact endocardial maps have been 


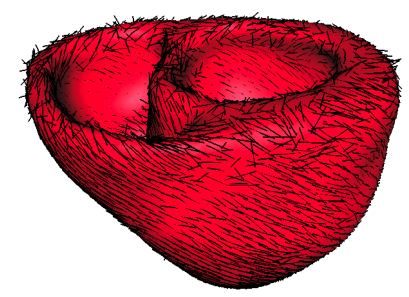

(a)

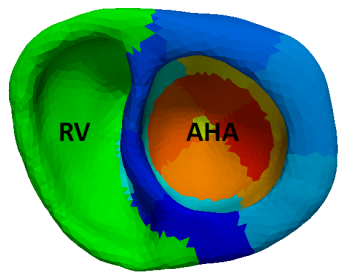

(b)

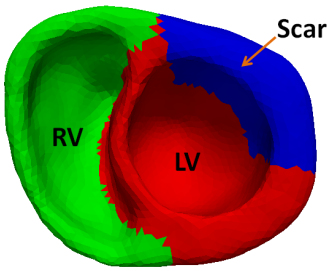

(c)

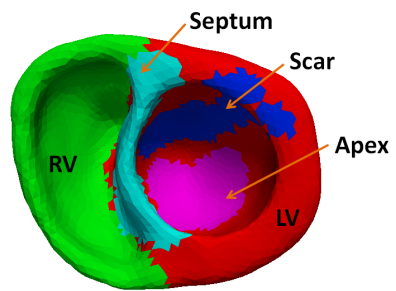

(d)

Figure 1: Heart representation. (a) Heart geometry and fiber orientations. (b) AHA-zone representation. (c) 3-zone representation. (d) 5-zone representation.

acquired at King's College London, St Thomas' Hospital during electrophysiology studies in an XMR suite (Rhode et al., 2005). Maps in sinus rhythm and during specific stimulation protocols were acquired, but only those in sinus rhythm were used as imaging can only be done in this rhythm. The extracted depolarization and repolarization isochrones then serve as input information to an electrophysiology personalization method (Relan et al., 2011) which minimizes the discrepancy between measured and simulated isochrones. Its output is a set of global parameters and local parameters (electrical conductivities) of the Mitchell-Schaeffer electrophysiology model (Mitchell and Schaeffer, 2003) which allow to interpolate, extrapolate and regularize the acquired isochrones. The personalized depolarization and repolarization times are used in (3) to control the active contraction force of the mechanical model.

\subsection{Kinematics Personalization}

Kinematics personalization consists in estimating the motion of cardiac structures from images. The same electromechanical model is used for both the kinematics personalization from cine MRI, and the mechanics personalization of estimating the biophysical parameters. This ensures the motion field produced by the kinematics personalization is consistent with the one required by the mechanics personalization, in terms of smoothness and spatial resolution. The kinematics personalization approach in Billet et al. (2009) is used with the cardiac electromechanical model in Section 2. Considering a twoventricular tetrahedral mesh constructed from an anatomical MRI (Figure 1), the evolution of the mesh is governed by (1) with an embedded image force:

$$
\mathbf{M U ̈}+\mathbf{C U}+\mathbf{K U}=\mathbf{F}_{b}+\mathbf{F}_{c}+\beta \mathbf{F}_{\mathrm{img}}
$$

In the kinematics personalization, $\mathbf{F}_{\text {img }}$ corresponds to a force vector which tracks salient image features in the MRI sequence, computed using a 3D block-matching algorithm to attract points towards the nearest edge voxels. To balance between model regularization and data attachment, we set $\beta$ based on a sensitivity analysis (Wong et al., 2010a), so that the personalized cardiac deformation is smooth while consistent with the apparent motion from the images. Image forces are not physiology based as their sole purpose is to help tracking the cardiac motion, and they are discarded during the mechanical personalization. The personalized nodal positions and velocities are obtained from the kinematics personalization, which are used as the inputs for contractility personalization, along with the personalized electrophysiology.

\section{Mechanics Personalization with Derivative-Free Optimiza- tion}

Kinematics personalization produces cardiac motion consistent with the apparent motion in an image sequence. Nevertheless, it cannot address the underlying physiological properties of the patient, such as the active contraction properties. To infer the physiological properties, mechanics personalization is required. Although a more complete mechanics personalization should address both active and passive parameters, here we only concentrate on the active parameters to reduce the complexity of the problem. To perform contractility personalization through variational data assimilation, an objective function and an optimization algorithm are required.

\subsection{Objective Function}

\subsubsection{Formulation}

The similarity between simulations and measurements is defined by an objective function. Supposing that the heart geometry is partitioned into regions, the objective function for variational data assimilation can be given as:

$$
\mathcal{F}(\boldsymbol{\theta})=\sum_{k} w_{k} \sum_{r} w_{r} \sum_{i}\left\|\overline{\mathbf{y}}_{k, r, i}-\mathbf{y}_{k, r, i}(\boldsymbol{\theta})\right\|^{2}
$$

where $\boldsymbol{\theta}$ is a vector comprising the parameters $\left(\sigma_{0}, \alpha_{c}, \alpha_{r}\right)$ of all regions. $\overline{\mathbf{y}}_{k, r, i}$ is the measurement at discrete time instant $k$ of point $i$ in region $r$, and $\mathbf{y}_{k, r, i}(\boldsymbol{\theta})$ is the corresponding simulated quantity. $w_{k}$ and $w_{r}$ are the weights of different time instants and regions.

The measurements $\overline{\mathbf{y}}_{k, r, i}$ are extracted from patient data, which are the personalized kinematic quantities in our case. Therefore, a time instant $k$ does not necessarily correspond to an image frame, but a time frame in the kinematics personalization. The weights $w_{k}$ and $w_{r}$ can be used to emphasize different time instants or regions. For example, if the contraction phase is of more interest, $w_{k}$ can be larger for the corresponding time instants. Similarly, regions with better measurements can have larger $w_{r}$. Nevertheless, as the optimal weights have not been studied and depend on applications and situations, they were set to one in the experiments. 

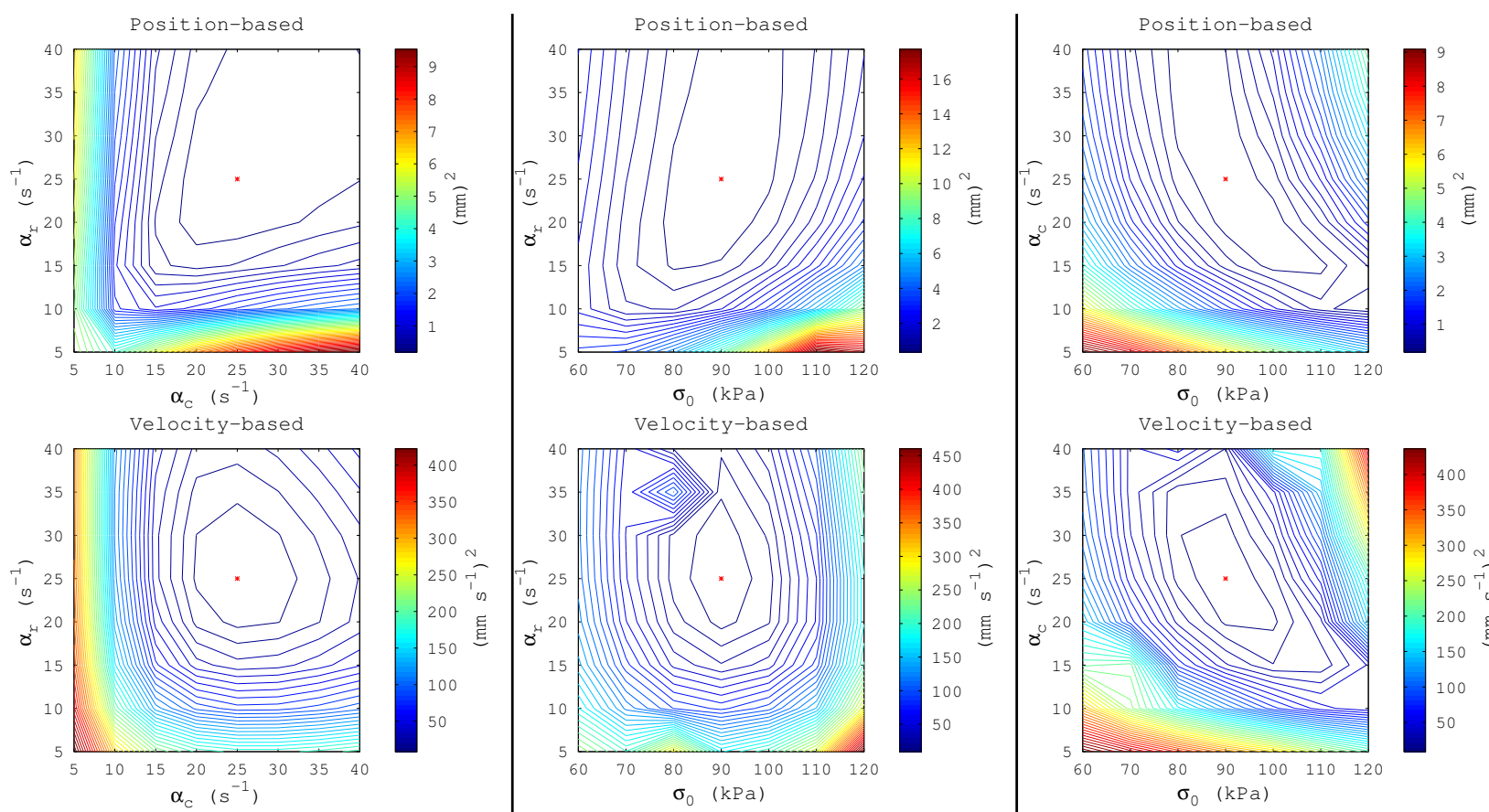

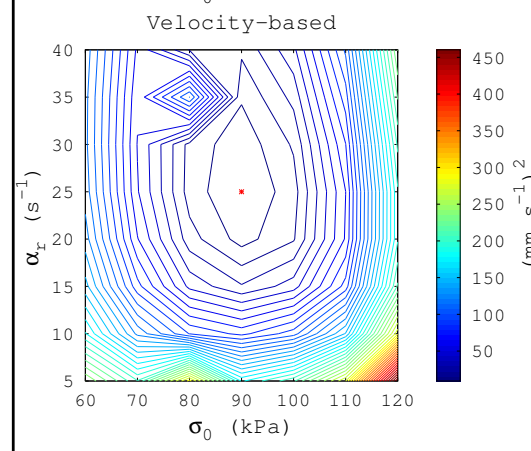

(b) $\alpha_{r}$ vs. $\sigma_{0}, \alpha_{c}=25 \mathrm{~s}^{-1}$

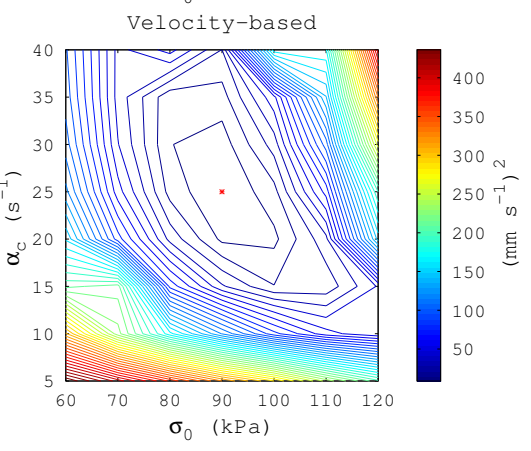

(c) $\alpha_{c}$ vs. $\sigma_{0}, \alpha_{r}=25 \mathrm{~s}^{-1}$

Figure 2: Landscapes of position-based and velocity-based objective functions, computed from simulated cardiac cycles. The red $*$ indicates the reference parameters $\left(\sigma_{0}=90 \mathrm{kPa}, \alpha_{c}=\alpha_{r}=25 \mathrm{~s}^{-1}\right)$. Each plot has 50 contours.

Different types of measurements $\overline{\mathbf{y}}_{k, r, i}$ can give different results. In Delingette et al. (2012); Sermesant et al. (2006b); Sundar et al. (2009), the positions of the personalized cardiac kinematics were used. As only the maximum contraction parameters $\sigma_{0}$ were estimated, the use of measured positions can provide meaningful results (see Section 5.2.1). Nevertheless, if the rates $\alpha_{c}$ and $\alpha_{r}$ are also desired, positions alone may not provide the necessary temporal information. Furthermore, it has been shown using control theory that velocity-based data assimilation can lead to a more stable system (Moireau et al., 2008). Therefore, we propose to use velocities instead.

To show the difference between using positions and velocities in (5), let $\Delta \mathbf{u}_{k, i}$ be the incremental displacement at time instant $k$ of point $i$ with time step $\Delta t_{k}$, where $\Delta t_{k}$ is the time step of numerical time integration when solving (1). Therefore, at time instant $\tilde{k}$, the velocity is $\mathbf{v}_{\tilde{k}, i}=\Delta \mathbf{u}_{\tilde{k}, i} / \Delta t_{\tilde{k}}$, and the position is $\mathbf{x}_{\tilde{k}, i}=\mathbf{x}_{0, i}+\sum_{k=1}^{\tilde{k}} \Delta \mathbf{u}_{k, i}$. Assuming the same time steps and initial positions are used by both personalized kinematics and simulation, the position difference gives:

$$
\overline{\mathbf{y}}_{\tilde{k}, i}-\mathbf{y}_{\tilde{k}, i}=\overline{\mathbf{x}}_{\tilde{k}, i}-\mathbf{x}_{\tilde{k}, i}=\sum_{k=1}^{\tilde{k}} \Delta \overline{\mathbf{u}}_{k, i}-\Delta \mathbf{u}_{k, i}
$$

which is actually the sum of incremental displacement differences of all previous time steps. Therefore, the temporal information cannot be properly reflected. On the other hand, the velocity difference gives:

$$
\overline{\mathbf{y}}_{\tilde{k}, i}-\mathbf{y}_{\tilde{k}, i}=\overline{\mathbf{v}}_{\tilde{k}, i}-\mathbf{v}_{\tilde{k}, i}=\frac{\Delta \overline{\mathbf{u}}_{\tilde{k}, i}-\Delta \mathbf{u}_{\tilde{k}, i}}{\Delta t_{\tilde{k}}}
$$

thus the temporal information is better preserved. In consequence, velocities are more preferable when $\alpha_{c}$ and $\alpha_{r}$ are desired.

\subsubsection{Landscape Investigation}

To further analyze the difference between position-based and velocity-based objective functions, a one-region case was investigated (Figure 2). Cardiac cycles were simulated on a patient heart geometry (see Section 5.1) with identical setting but different values of $\sigma_{0}, \alpha_{c}$, and $\alpha_{r}$. The heart geometry was treated as one region, and a total of 448 simulations were performed $\left(\sigma_{0}: 60-120 \mathrm{kPa}\right.$ with stride $=10 \mathrm{kPa} ; \alpha_{c}$ and $\alpha_{r}: 5-40$ $\mathrm{s}^{-1}$ with stride $\left.=5 \mathrm{~s}^{-1}\right)$. By using one of the simulations as a reference $\left(\sigma_{0}=90 \mathrm{kPa}, \alpha_{c}=\alpha_{r}=25 \mathrm{~s}^{-1}\right)$, the $3 \mathrm{D}$ objective functions were computed. To visualize the $2 \mathrm{D}$ landscapes, the cutting planes centering at the reference parameters are shown.

Figure 2 shows that the velocity-based objective function has better landscapes than the position-based one. The positionbased landscapes have relatively flat valleys, thus the minimum is more difficult to be identified as the values around are similar. Figure 2(a) shows that the velocity-based objective function is more capable of identifying contraction and relaxation rates. Figure 2(b) and (c) show that both objective functions have similar capability of identifying the maximum contraction parameter, as they have similar changes in the $\sigma_{0}$ direction. Nevertheless, the position-based objective function is less capable of identifying the rate parameters as there are long valleys in the $\alpha_{c}$ and $\alpha_{r}$ directions. These observations are consistent with the explanations in the previous section that the rate information is better preserved with the velocity-based objective function. 

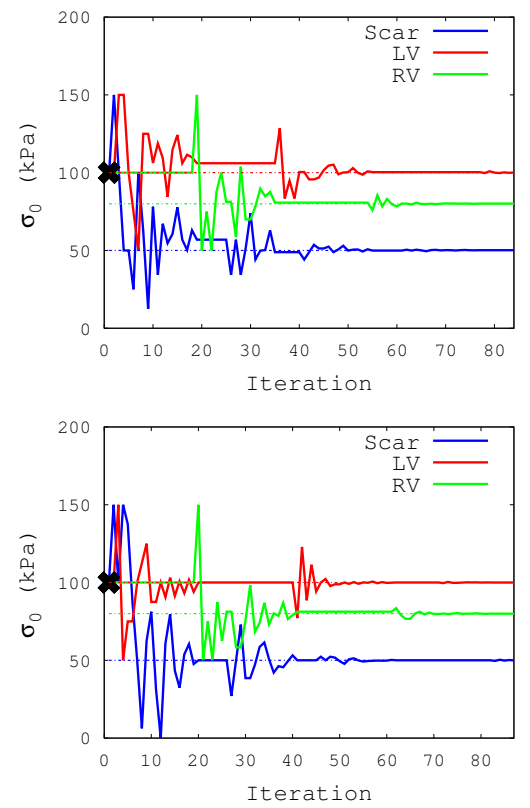

(a) 3-zone
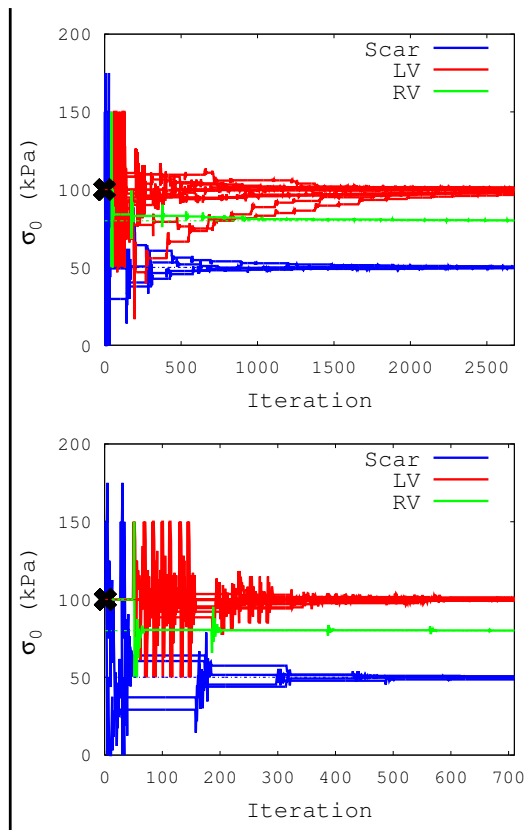

(b) AHA-zone
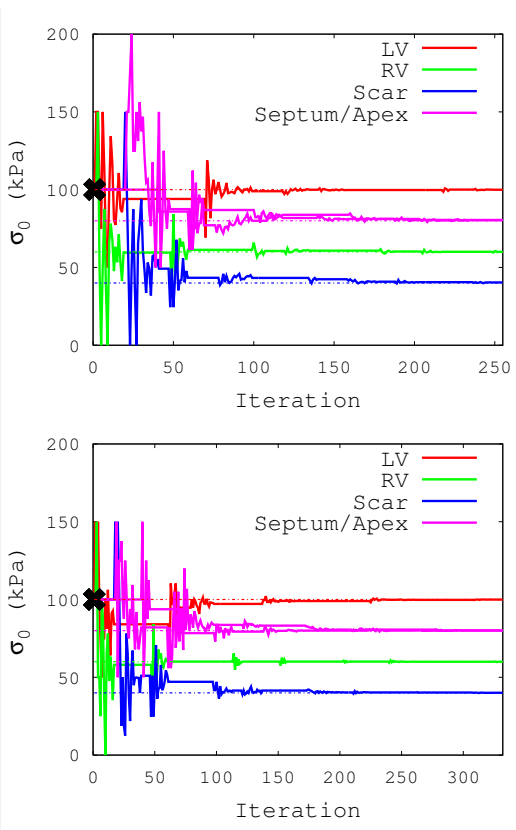

(c) 5-zone

Figure 3: Synthetic data. Estimations of maximum contraction parameters $\left(\sigma_{0}\right)$ only, with the contraction and relaxation rates fixed at the ground truth values. The dotted lines represent the ground truth values, and the black crosses represent the initial parameters. Top: position-based optimization. Bottom: velocity-based optimization. In the legend of (b), Scar corresponds to AHA zone 4, 5, 10, and 11; LV corresponds to AHA zone 1-3, 6-9, and 12-17.

\subsection{Derivative-Free Optimization}

To estimate the contraction parameters, the optimization problems were solved using gradient-based algorithms on synthetic data in Sermesant et al. (2006b); Sundar et al. (2009), and on clinical data in Delingette et al. (2012). In Delingette et al. (2012), the gradient-based quasi-Newton L-BFGS-B algorithm was used. As the model involves interactions between myocardial deformation, ventricular pressures, contraction stresses, and different boundary conditions, it is difficult to compute the gradient analytically without making significant simplifications which sacrifice the model integrity and thus the estimation accuracy. In consequence, analytical gradient evaluation may limit the flexibility of choosing the appropriate objective function, and the accuracy and stability may be reduced if improper simplifications are made (Conn et al., 2009).

On the other hand, although finite difference is a popular numerical alternative when analytical evaluation of the gradient is infeasible (Rao, 2009), the associated computational complexity is impractical to our problem. Let $n$ be the number of parameters to be estimated. $n+1$ function evaluations are required for the forward or backward approximations, and $2 n+1$ function evaluations are required for the more accurate central difference approximation. As each function evaluation involves the simulation of the whole cardiac cycle, finite difference is impractical. Furthermore, finite difference is sensitive to the step size and the noisiness of the objective function (Rao, 2009). Therefore, it is not the optimal choice to our problem.

Some optimization methods assume that the objective function is locally quadratic, such as the BFGS and the BOBYQA algorithms (Powell, 2003). These methods can be fast and accurate when the locally quadratic assumption is satisfied. Never- theless, as our objective functions do not fulfill this requirement (Figure 2), the use of these methods may lead to suboptimal results.

\subsubsection{The Subplex Method}

With respect to these issues, direct search methods which make few assumptions about the objective function are more preferable, and the subplex method (SUBspace-searching simPLEX, SUBPLEX) is adopted (Rowan, 1990). SUBPLEX is a generalization of the Nelder-Mead simplex method (NMS) (Nelder and Mead, 1965), a popular method for optimizing noisy functions. A simplex in $n$-dimensional space is a convex hull of $n+1$ points, for example, a triangle in 2D and a tetrahedron in 3D. In NMS, a simplex moves through the objective function space, changing size and shape, and shrinking near the minimum. NMS performs well when the dimension is small (e.g. $\leq 5$ ), but can be inefficient when the dimension is much higher (Rowan, 1990). To address this issue, SUBPLEX decomposes the high-dimensional space into subspaces that NMS can search efficiently. In each optimization cycle, given the minimum and maximum subspace dimensions, SUBPLEX determines the number of subspaces and the dimensions and directions of the subspaces, and then each subspace is minimized using NMS. To efficiently generate an improved set of subspaces after each cycle, SUBPLEX chooses subspaces so that the direction of progress approximately lies in the first subspace. Therefore, if the minimum and maximum subspace dimensions equal $n$, SUBPLEX is equivalent to NMS. On the other hand, if the subspace dimensions equal one, SUBPLEX becomes an alternating variable method. For more details please refer to Section 5.3 in (Rowan, 1990). 

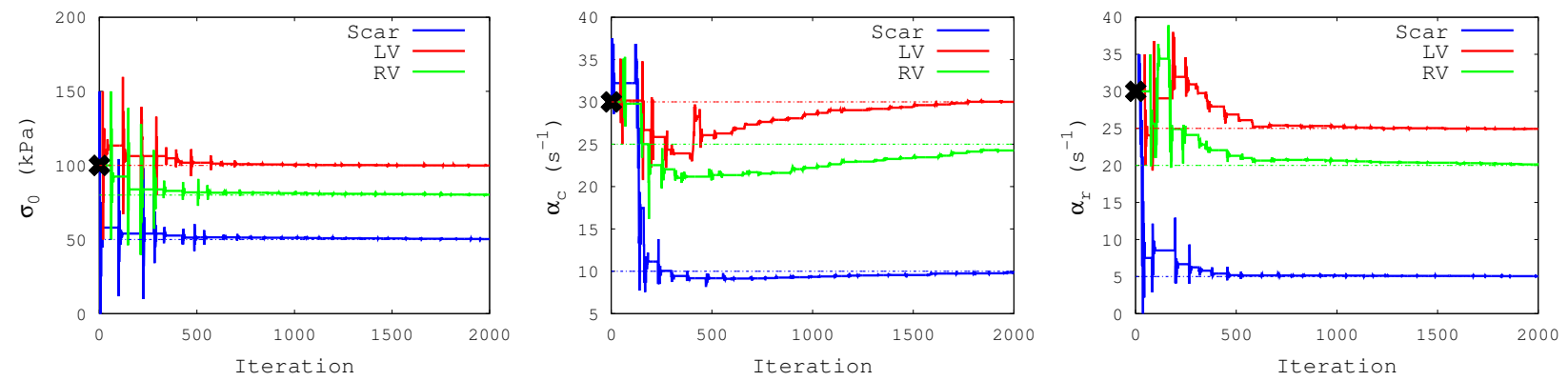

(a) Position-based optimization.
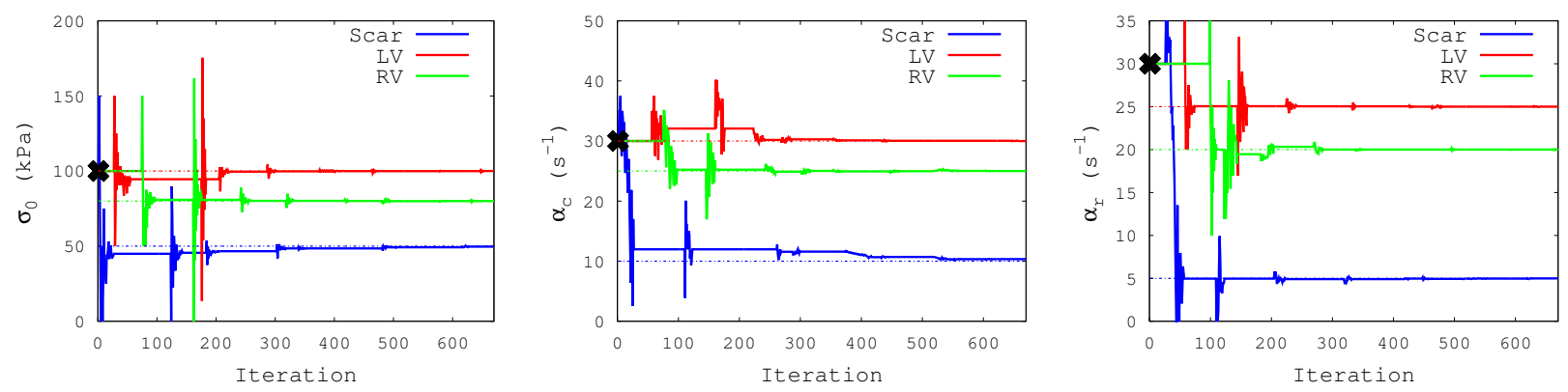

(b) Velocity-based optimization

Figure 4: Synthetic data. Estimations of 3-zone parameters, with the dotted lines representing the ground truth values, and the black crosses representing the initial parameters. Left to right: maximum contraction parameter $\sigma_{0}$, contraction rate $\alpha_{c}$, and relaxation rate $\alpha_{r}$.

Table 1: Young's moduli $\left(E_{f}, E_{c f}\right)$ and Poisson's ratios $\left(\nu_{f}, \nu_{c f}\right)$ along and across fiber (Glass et al., 1991).

\begin{tabular}{|c|c|c|c|}
\hline$E_{f}$ & $E_{c f}$ & $\nu_{f}$ & $\nu_{c f}$ \\
\hline $75 \mathrm{kPa}$ & $25 \mathrm{kPa}$ & 0.47 & 0.47 \\
\hline
\end{tabular}

Table 2: Synthetic data. Ground truth parameters.

\begin{tabular}{|c|c|c|c|}
\hline 3-zone & LV & RV & Scar \\
\hline$\sigma_{0}(\mathrm{kPa})$ & 100 & 80 & 50 \\
\hline$\alpha_{c}\left(\mathrm{~s}^{-1}\right)$ & 30 & 25 & 10 \\
\hline$\alpha_{r}\left(\mathrm{~s}^{-1}\right)$ & 25 & 20 & 5 \\
\hline
\end{tabular}

\begin{tabular}{|c|c|c|c|c|c|}
\hline 5-zone & LV & RV & Scar & Septum & Apex \\
\hline$\sigma_{0}(\mathrm{kPa})$ & 100 & 60 & 40 & 80 & 80 \\
\hline$\alpha_{c}\left(\mathrm{~s}^{-1}\right)$ & 30 & 20 & 15 & 25 & 25 \\
\hline$\alpha_{r}\left(\mathrm{~s}^{-1}\right)$ & 25 & 15 & 10 & 20 & 20 \\
\hline
\end{tabular}

\section{Evaluation on Synthetic Data}

To evaluate the performance of our framework, experiments were performed on synthetic data. The comparisons between the position-based and velocity-based objective functions, the sensitivities of the velocity-based framework with respect to measurement noise and initial parameters, and also the comparisons with the L-BFGS-B and BOBYQA optimization algorithms, are presented.

\subsection{Experimental Setups}

The heart representation was created from the data of a patient with myocardial infarction. The heart geometry was segmented from the image frame at mid-diastole, and a FEM mesh with synthetic fiber orientations was obtained (Figure 1(a)). The personalized $T_{d}$ and $T_{r}$ derived from the patient noncontact endocardial electrical maps were used in (3) (Relan et al., 2011). The passive mechanical parameters used are shown in Table 1. To show that our framework can be used with different types of zone partitioning, three settings were tested:

1. AHA-zone representation (Figure 1(b)): the American Heart Association (AHA) nomenclature (Cerqueira et al., 2002) was used for the left ventricle. The right ventricle is one zone $(\mathrm{RV})$.

2. 3-zone representation (Figure 1(c)): AHA zone 4, 5, 10, and 11 were grouped into one zone (Scar), and the remaining AHA zones were grouped into another zone (LV). The right ventricle is one zone (RV).

3. 5-zone representation (Figure 1(d)): the infarcted region was identified from late enhancement MRI (Scar). The rest of the left ventricle was partitioned into LV, Septum, and Apex. The right ventricle is one zone (RV).

Two simulations with active contraction parameters listed in Table 2 were performed on the 3-zone and 5-zone representations to provide the ground truth measurements. To emulate the condition of using real data, only the positions and velocities on the heart surfaces were used for the parameter estimations. The AHA-zone representation was only used for the parameter estimation with the 3-zone measurements to verify if the framework is capable of estimating high-dimensional parameters. The initial parameters were $\sigma_{0}=100 \mathrm{kPa}, \alpha_{c}=\alpha_{r}=30$ $\mathrm{s}^{-1}$, which were used in all experiments in this paper (except the initial parameter tests). As the objective functions are relatively flat around the optimal points, to avoid immature convergence, small absolute parameter tolerances were used as the 

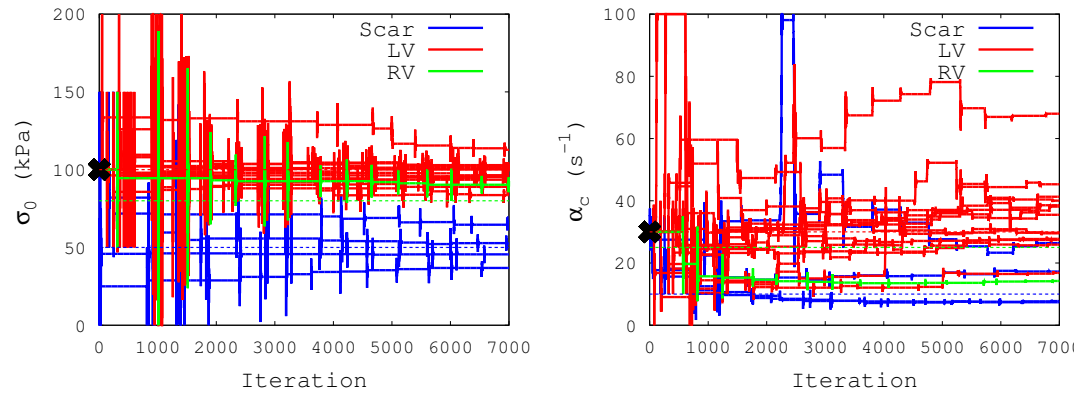

(a) Position-based optimization.

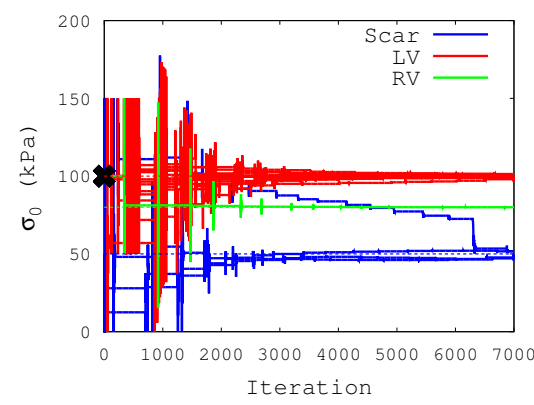

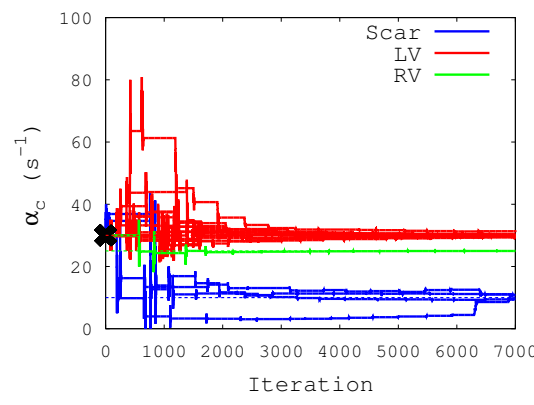

(b) Velocity-based optimization.
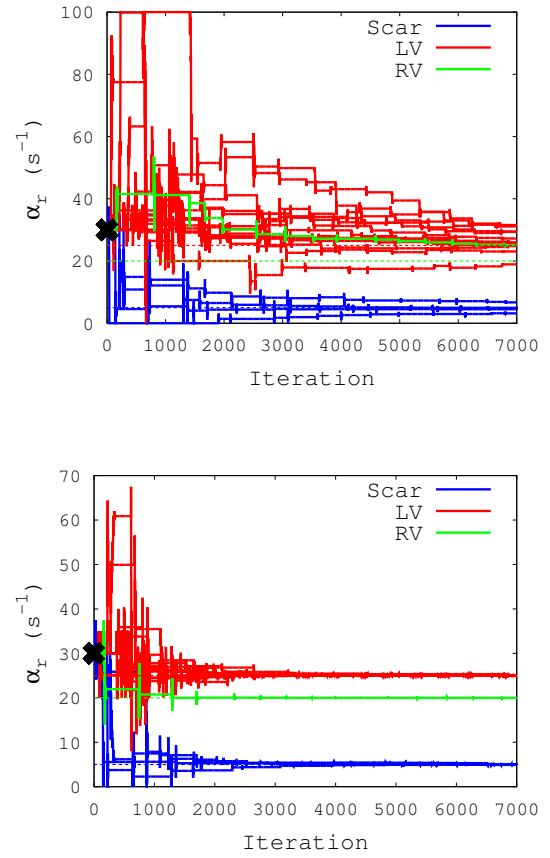

Figure 5: Synthetic data. Estimations of AHA-zone parameters, with the dotted lines representing the ground truth values, and the black crosses representing the initial parameters. Left to right: maximum contraction parameter $\sigma_{0}$, contraction rate $\alpha_{c}$, and relaxation rate $\alpha_{r}$. In the legend, Scar corresponds to AHA zone 4, 5, 10, and 11; LV corresponds to AHA zone 1-3, 6-9, and 12-17.

convergence criteria, with $\sigma_{0}=0.5 \mathrm{kPa}, \alpha_{c}=\alpha_{r}=0.05 \mathrm{~s}^{-1}$. The maximum numbers of iterations of the 3-zone, 5-zone, and AHA-zone estimations were 2000, 5000, and 7000 respectively, which were chosen empirically. Each iteration corresponds to a simulation of the cardiac cycle.

\subsection{Results and Discussion}

\subsubsection{Comparisons between Position-Based and Velocity-Based Optimizations}

Figure 3 shows the estimations of the maximum contraction parameters $\left(\sigma_{0}\right)$, with the contraction and relaxation rates fixed at the ground truth values. Both position-based and velocitybased frameworks obtained parameters very close to the ground truth, although the number of iterations of the position-based framework is about three times as that of the velocity-based framework in the AHA-zone setting. This means that the frameworks are similar in accuracy if only the maximum contraction parameters are of interest.

Figure 4 shows the estimations of 3-zone parameters, and both position-based and velocity-based optimizations have good results. Nevertheless, the velocity-based optimization converged after 670 iterations, while the position-based optimization did not converge with the maximum number of iterations (2000).

To verify the capability of estimating high-dimensional parameters, estimations were performed using the AHA-zone representation with the 3-zone measurements (Figure 5). Both optimizations did not converge with the maximum number of iterations (7000), which may be caused by the relatively high searching dimension $(n=54)$, or by the non-uniqueness of the solution. Nevertheless, the velocity-based optimization obtained much better results. This is consistent with the observation in Figure 2 that the position-based objective function has a larger flat region, which effect is amplified in the highdimensional parameter estimation.

To show that the framework can be applied to different types of zone partitioning, experiments were performed on the 5-zone representation (Figure 6). Similar to the AHA-zone estimation, the velocity-based optimization has much better results, and converged after 2724 iterations. On the other hand, the position-based optimization did not converge within the maximum number of iterations (5000).

Figure 7 shows the mean position differences between the ground truth and the personalized simulations of all nodes in a cardiac cycle. For the 3-zone optimization, the mean position differences of both position-based and velocity-based optimizations are too small $(<0.05 \mathrm{~mm})$ to be meaningfully compared. For the AHA-zone and 5-zone optimizations, the velocity-based optimizations are much better. Especially for the 5-zone optimization, the standard deviation of the velocity-based estimation is too small to be visualized when put together with the position-based optimization. All of these show that the velocity-based optimization is better than the position-based optimization for simultaneous estimation of maximum contraction parameters and contraction and relaxation rates.

\subsubsection{Sensitivities to Noise and Initial Parameters}

To verify the sensitivity of the framework with respect to measurement noise, the simulated 3-zone nodal velocity measurements were added with zero-mean noises of different signal- 

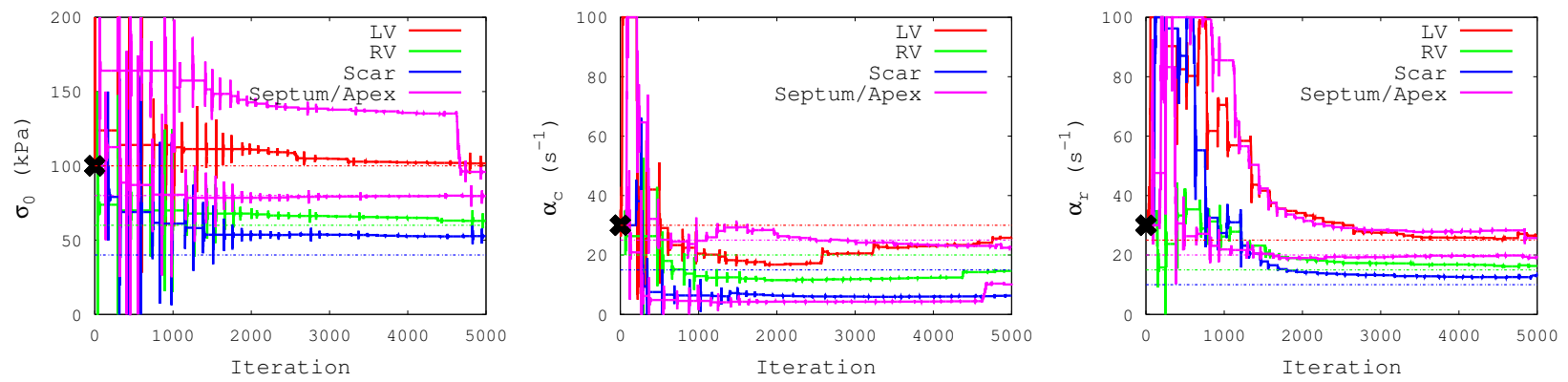

(a) Position-based optimization.
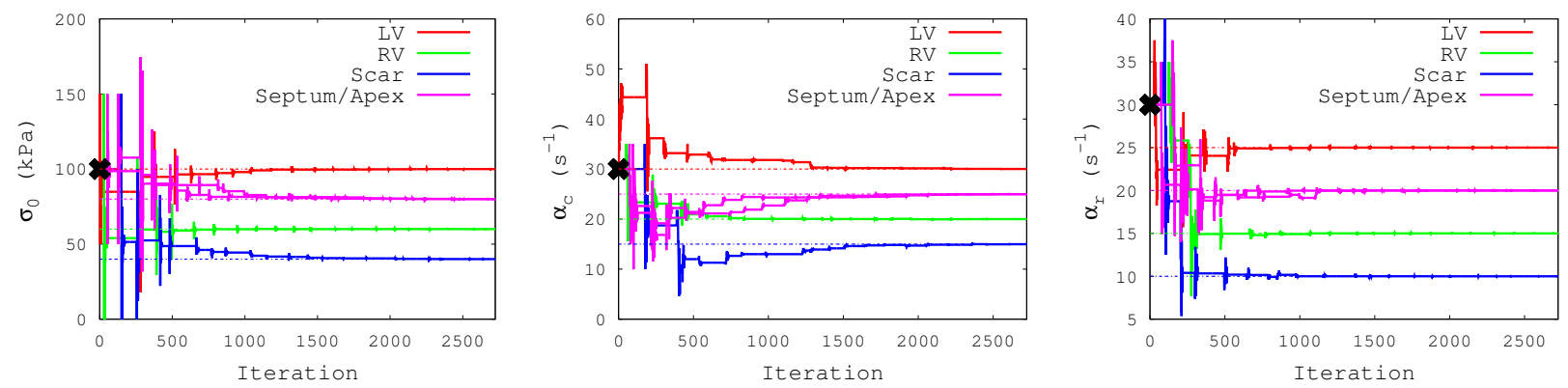

(b) Velocity-based optimization

Figure 6: Synthetic data. Estimations of 5-zone parameters, with the dotted lines representing the ground truth values, and the black crosses representing the initial parameters. Left to right: maximum contraction parameter $\sigma_{0}$, contraction rate $\alpha_{c}$, and relaxation rate $\alpha_{r}$.

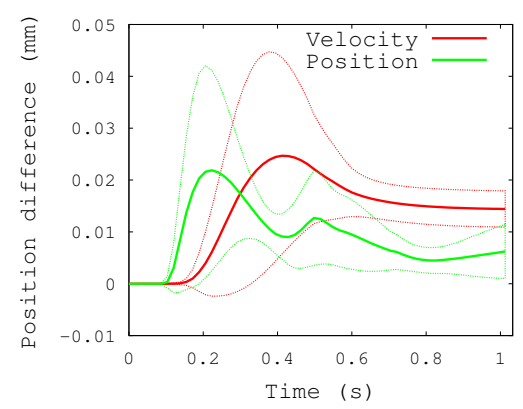

(a) 3-zone.

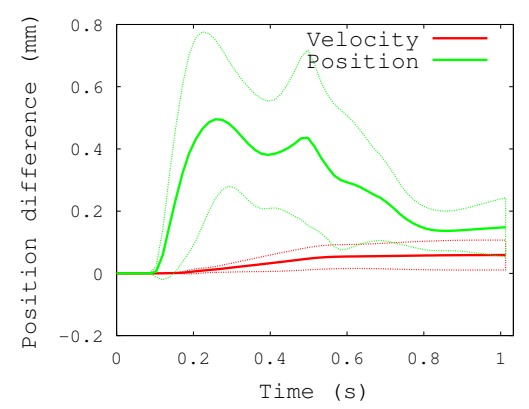

(b) AHA-zone.

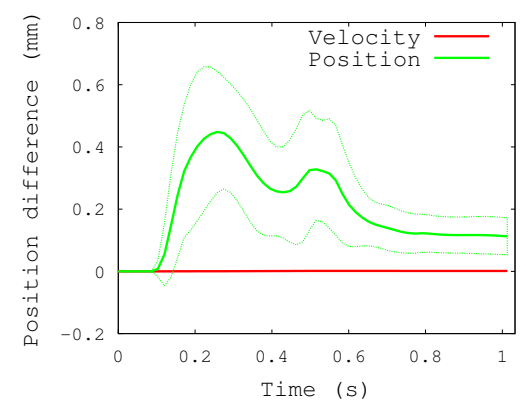

(c) 5-zone.

Figure 7: Synthetic data. Mean position differences of all nodes between the ground truth and the personalized simulations in a cardiac cycle. The dotted lines envelop the standard deviations.

to-noise ratios (SNR $=1,2$, and 5), and the velocity-based optimizations were performed. Table 3 shows that the estimated parameters are almost identical to the ground truth, even in the worst case when the $\mathrm{SNR}=1$. One reason of such robustness is the use of variational data assimilation. In fact, the smoothness of the objective function (5) is independent of the measurements, as they are the same at any $\boldsymbol{\theta}$. Therefore, the effects of zero-mean noises to the estimation should be minimal. This can be shown by the similar numbers of iterations $(654,649$, and 665 , for $\mathrm{SNR}=1,2$, and 5, respectively).

To verify the sensitivity of the framework with respect to different initial parameters, 3-zone velocity-based optimizations were performed with different initializations (Case 1: $\sigma_{0}=50$ $\mathrm{kPa}, \alpha_{c}=\alpha_{r}=5 \mathrm{~s}^{-1}$; Case 2: $\sigma_{0}=50 \mathrm{kPa}, \alpha_{c}=\alpha_{r}=30$ $\mathrm{s}^{-1}$; Case 3: $\sigma_{0}=100 \mathrm{kPa}, \alpha_{c}=\alpha_{r}=5 \mathrm{~s}^{-1}$; Case 4: $\sigma_{0}=100 \mathrm{kPa}, \alpha_{c}=\alpha_{r}=30 \mathrm{~s}^{-1}$ ). Table 4 shows that the framework is robust to initializations, which means that the subplex algorithm matches the characteristics of the objective function. The number of iterations from Case 1 to 4 are 729 , 694,980 , and 670 respectively.

\subsubsection{Comparisons to L-BFGS-B and BOBYQA Optimization Algorithms}

To show the importance of using the derivative-free SUBPLEX algorithm, the 3-zone velocity-based optimizations were performed using the L-BFGS-B and the BOBYQA algorithms.

For the L-BFGS-B algorithm, as the analytical gradient of the objective function is very difficult to derive, the forward finite difference approximation was used. This requires $n+1$ function evaluations for each gradient computation, with $n(=$ $9)$ the total number of parameters. As mentioned in the literature (Rao, 2009), finite difference approaches may introduce 
Table 3: Synthetic data. Velocity-based estimations of 3-zone parameters with different SNR. See Table 2 for the ground truth values.

\begin{tabular}{|c|c|c|c|c|c|c|c|c|c|}
\hline SNR & \multicolumn{3}{|c|}{1} & \multicolumn{3}{c|}{2} & \multicolumn{3}{|c|}{5} \\
\hline Zone & LV & RV & Scar & LV & RV & Scar & LV & RV & Scar \\
\hline$\sigma_{0}(\mathrm{kPa})$ & 100.0 & 80.0 & 50.3 & 100.0 & 80.1 & 50.2 & 100.0 & 80.1 & 49.5 \\
\hline$\alpha_{c}\left(\mathrm{~s}^{-1}\right)$ & 30.2 & 24.9 & 10.0 & 29.9 & 25.0 & 9.9 & 30.0 & 25.0 & 10.4 \\
\hline$\alpha_{r}\left(\mathrm{~s}^{-1}\right)$ & 24.9 & 20.0 & 4.9 & 25.0 & 20.1 & 5.0 & 25.0 & 20.0 & 4.9 \\
\hline
\end{tabular}

Table 4: Synthetic data. Velocity-based estimations of 3-zone parameters with different initializations $\left(\mathrm{Case} 1: \sigma_{0}=50 \mathrm{kPa}, \alpha_{c}=\alpha_{r}=5 \mathrm{~s}-1 ;\right.$ Case $2: \sigma_{0}=50$ $\mathrm{kPa}, \alpha_{c}=\alpha_{r}=30 \mathrm{~s}^{-1}$; Case 3: $\sigma_{0}=100 \mathrm{kPa}, \alpha_{c}=\alpha_{r}=5 \mathrm{~s}^{-1}$; Case 4: $\sigma_{0}=100 \mathrm{kPa}, \alpha_{c}=\alpha_{r}=30 \mathrm{~s}^{-1}$ ). See Table 2 for the ground truth values.

\begin{tabular}{|c|c|c|c|c|c|c|c|c|c|c|c|c|}
\hline Case & \multicolumn{3}{|c|}{1} & \multicolumn{3}{|c|}{2} & \multicolumn{3}{|c|}{3} & \multicolumn{3}{|c|}{4} \\
\hline Zone & LV & RV & Scar & LV & RV & Scar & LV & RV & Scar & LV & RV & Scar \\
\hline$\sigma_{0}(\mathrm{kPa})$ & 100.0 & 80.0 & 49.9 & 100.0 & 80.0 & 50.0 & 100.0 & 80.0 & 50.0 & 100.0 & 80.0 & 49.7 \\
\hline$\alpha_{c}\left(\mathrm{~s}^{-1}\right)$ & 30.0 & 25.0 & 10.1 & 30.0 & 25.0 & 10.0 & 30.0 & 25.0 & 10.0 & 30.0 & 25.0 & 10.3 \\
\hline$\alpha_{r}\left(\mathrm{~s}^{-1}\right)$ & 25.0 & 20.0 & 5.0 & 25.0 & 20.0 & 5.0 & 25.0 & 20.0 & 5.0 & 25.0 & 20.0 & 5.0 \\
\hline
\end{tabular}

numerical difficulties regarding the step size. Among the different tested step sizes, not all of them can lead to successful optimization because of numerical errors. The results in Figure 8(a) represent the best results among the successful tests, with the step size of $\sigma_{0}$ as $1 \mathrm{kPa}$, and that of $\alpha_{c}$ and $\alpha_{r}$ as $0.1 \mathrm{~s}^{-1}$. Note that because of the use of finite difference approximation, the number of function evaluations is ten times of the number of iterations.

Figure 8 shows that for the maximum contraction parameters, both L-BFGS-B and BOBYQA algorithms can provide fairly good estimations. Nevertheless, they cannot properly estimate the contraction and relaxation rates. In fact, using other initial parameters can provide better results, but this means that these two algorithms are sensitive to initial parameters with the proposed objective function. These observations are consistent with the limitations described in Section 4.2 that, both L-BFGS$\mathrm{B}$ and BOBYQA algorithms assume locally quadratic objective functions, which is not the case of the proposed objective function with respect to the rate parameters.

Figure 9 shows that among the tested optimization algorithms, the personalized simulation of the L-BFGS-B algorithm deviates most from the ground truth, while that of the BOBYQA algorithm is slightly better. The corresponding deviation of the SUBPLEX algorithm is almost nil when compared with the others.

\section{Evaluation on Clinical Data}

To verify the clinical applicability of the proposed framework, experiments were performed on clinical data from patients and healthy volunteers. As ground truth parameters are unavailable for clinical data, numerical comparisons are impossible. Therefore, these experiments are to validate, in terms of the consistency with the personalized kinematics and invasive measurements, if the personalization framework can provide parameters reflecting the actual physiology of the subject. As we have shown that the velocity-based framework outperforms the position-based framework in synthetic data, we only concentrate on the velocity-based framework in the following discussions.

\subsection{Experimental Setups}

\subsubsection{Patients with Cardiac Diseases}

Three data sets were tested. Patient 1 has myocardial infarction, whose data set was used in Section 5.1 to generate the synthetic data, with the infarcted regions identified through late enhancement MRI. Patient 2 and 3 have dilated cardiomyopathy but without identified infarctions. All patients have left bundle branch block (LBBB) and suffer from heart failure. The numbers of frames of Patient 1, 2, and 3 are 30 (1.03 s), 30 (0.73 $\mathrm{s})$, and 40 (1.07 s) respectively. The corresponding in-plane resolutions are $1.56,1.45$, and $1.52 \mathrm{~mm}^{2}$, and all images have 10 $\mathrm{mm}$ slice thickness. All data sets have the endocardial activation maps measured with the Ensite balloon (St. Jude Medical, $\mathrm{MN}$ ), which were extrapolated to the myocardial volume using an electrophysiological model to provide the subject-specific $T_{d}$ and $T_{r}$ in (3) for the experiments (Relan et al., 2011). The left-ventricular blood pressures were also measured invasively.

For each data set, the heart geometry was segmented from the image frame at mid-diastole, and a FEM mesh with synthetic fiber orientations was constructed (Sermesant et al., 2012). For Patient 1, the 5-zone heart representation in Section 5.1 was used (Figure 1 (d)) with the known scar region. For Patient 2 and 3, AHA-zone representations similar to that in Figure 1 (b) were used. Kinematics personalizations were performed to provide the nodal velocities for the experiments, and only the nodes on the heart surfaces were used as motion information is unavailable inside the myocardium for cine MRI. The performance and noise-handling capability of the kinematics personalization have been studied in Billet et al. (2009); Sermesant et al. (2009); Wong et al. (2010a). The same passive mechanical parameters, initial parameters, and convergence criteria in Section 5.1 were applied. The maximum number of iterations was 5000 .

\subsubsection{Healthy Volunteers}

To show that our framework can also provide results reflecting healthy cardiac conditions, experiments were performed on volunteers' data without known cardiac diseases. Each data set has a cine MRI sequence of 30 frames, with the heart periods of Volunteer 1, 2, and 3 as $0.83,0.87$, and 1.2 s respectively. All data sets have $1.25 \mathrm{~mm}^{2}$ in-plane resolution and $10 \mathrm{~mm}$ slice 

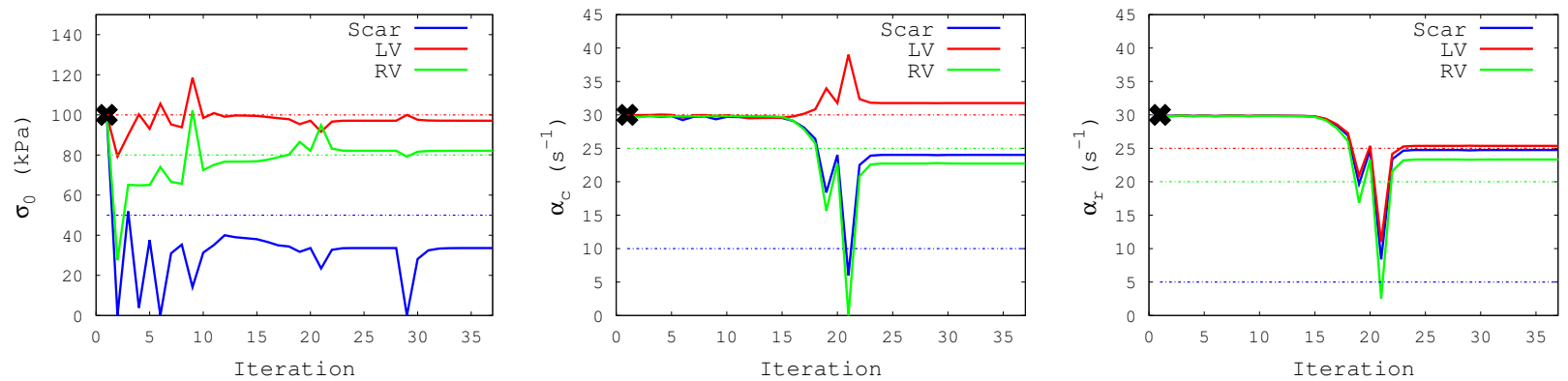

(a) L-BFGS-B.
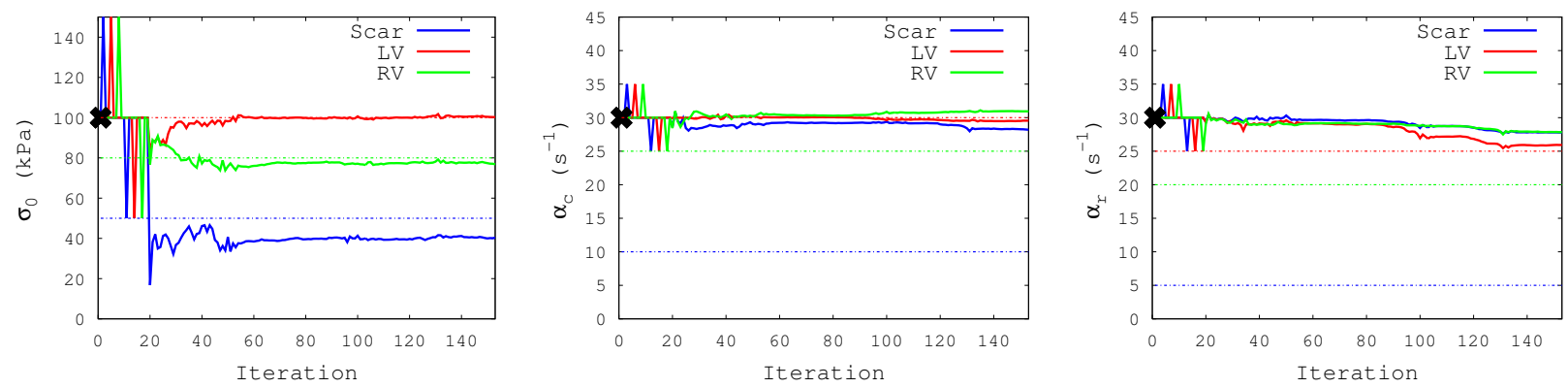

(b) BOBYQA.

Figure 8: Synthetic data. Velocity-based estimations of 3-zone parameters using the L-BFGS-B and BOBYQA algorithms, with the dotted lines representing the ground truth values, and the black crosses representing the initial parameters. Left to right: maximum contraction parameter $\sigma_{0}$, contraction rate $\alpha_{c}$, and relaxation rate $\alpha_{r}$.

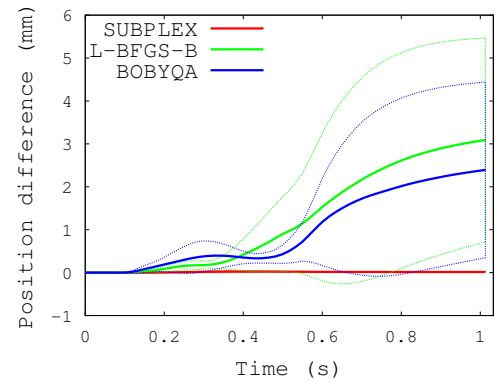

Figure 9: Synthetic data. Mean position differences of all nodes between the ground truth and the personalized simulations in a cardiac cycle. The dotted lines envelop the standard deviations.

thickness. Neither endocardial activation maps nor ventricular blood pressures were measured. The electrical propagations were obtained using simulations with normal parameters (Sermesant et al., 2006a).

Each heart geometry was segmented from the image frame at mid-diastole, and a FEM mesh with synthetic fiber orientations was constructed. AHA-representations were used. Kinematics personalizations were performed to provide the surface velocities for the experiments. The same optimization settings as the patient data sets were applied.

\subsection{Results and Discussion}

Figure 10 and 11 provide the comparisons among the personalized kinematics, simulations with initial parameters, and personalized simulations, for the patient and volunteer data re- spectively. They also show the active contraction stresses at the end of systole. For the patient data (Figure 10), in all cases, the personalized simulations are much closer to the personalized kinematics, showing large improvements from the initializations. For Patient 1, the scar region has low contractility, which is consistent to the pathology. On the other hand, the septum and apex also have low contractility even they are not infarcted. The low contractility at the septum is related to the septal flash of the patient, which is due to the difficulty of the septum to properly contract at the right timing. As this is a complicated phenomenon which requires more detailed electrophysiological model, mechanical model, and their corresponding parameter estimation, it cannot be accounted by our current framework. For the apex, as it is located outside the image region, the estimated contractility cannot be justified.

Similarly, Figure 11 shows improvements of the personalized simulations on the volunteer data, but the improvements are much less than those of the patient data. This is because the initial parameters account for the normal conditions, and thus the initial simulations are already well matched to the personalized kinematics. For the personalized simulations, the left ventricles of the patients contract much less than those of the volunteers, which is consistent with the dilated cardiomyopathy of the patients.

To further show the realism of the personalized mechanics, the simulated ventricular blood pressures through the ventricular isovolumetric constraint and the three-element Windkessel model (see Section 2) are compared with those invasively measured from the patients (Figure 12). The same Windkessel 


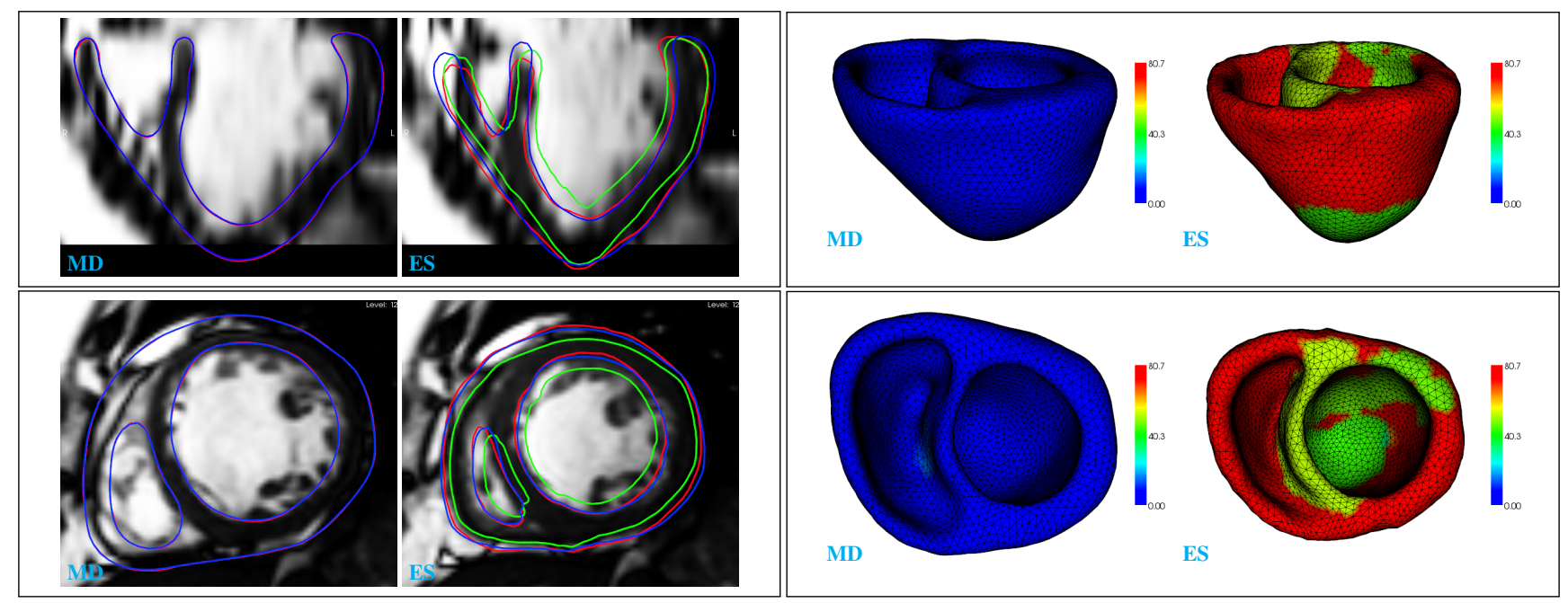

(a) Patient 1.

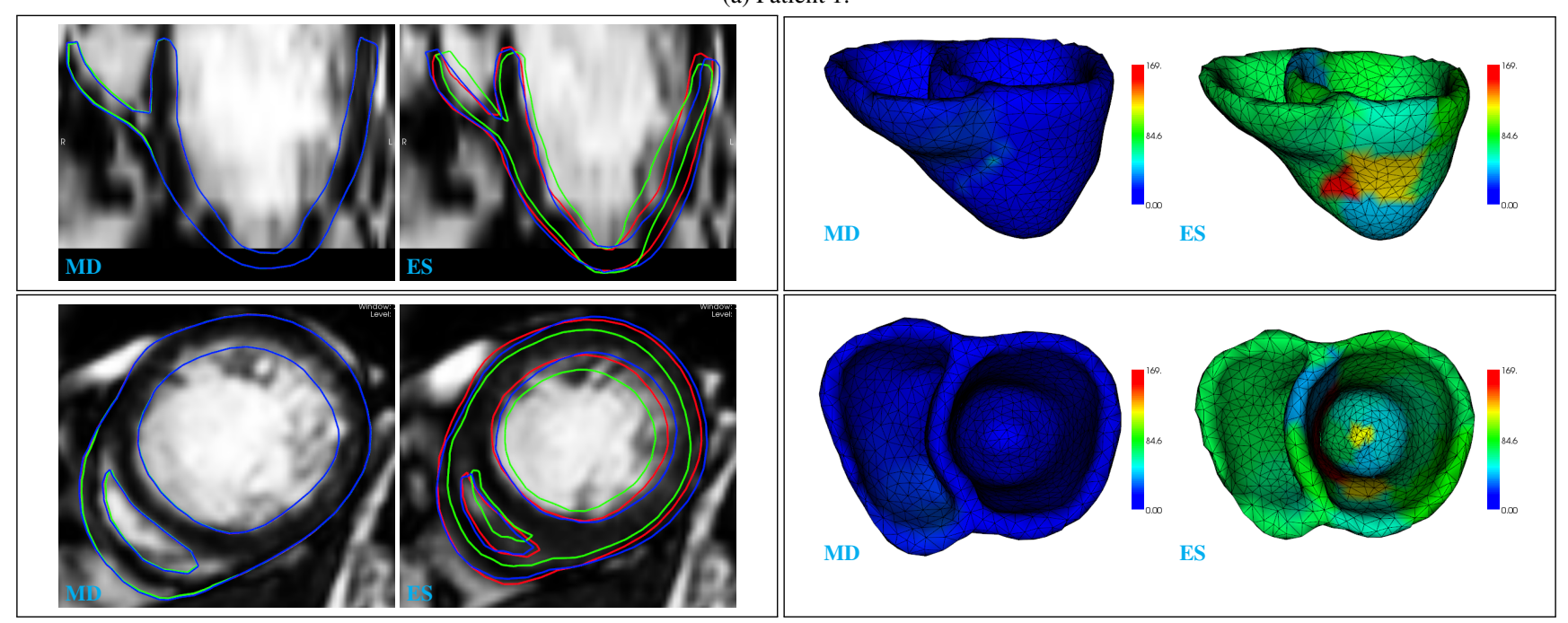

(b) Patient 2.

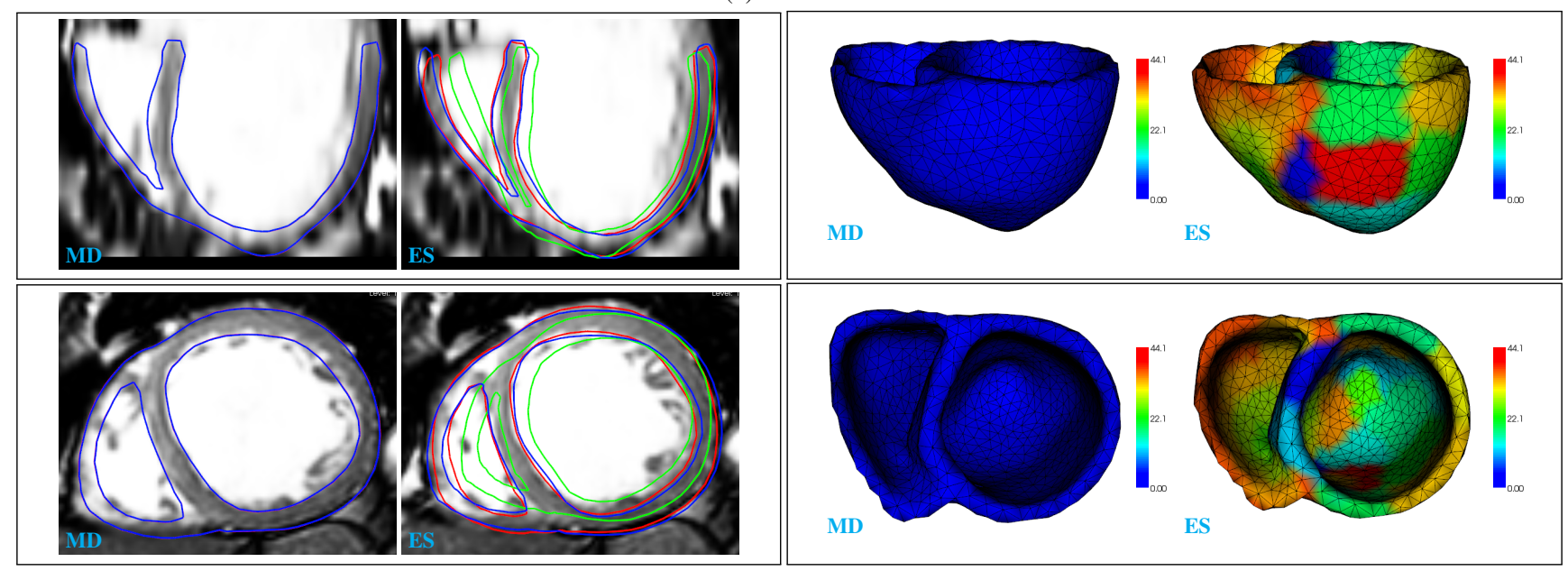

(c) Patient 3.

Figure 10: Patient data. Results at mid diastole (MD) and end systole (ES). Left: heart geometries overlapped with images, with red, green, and blue representing personalized kinematics, initializations, and personalized simulations respectively. Right: personalized simulations and the corresponding contraction stresses in $\mathrm{kPa}$. 


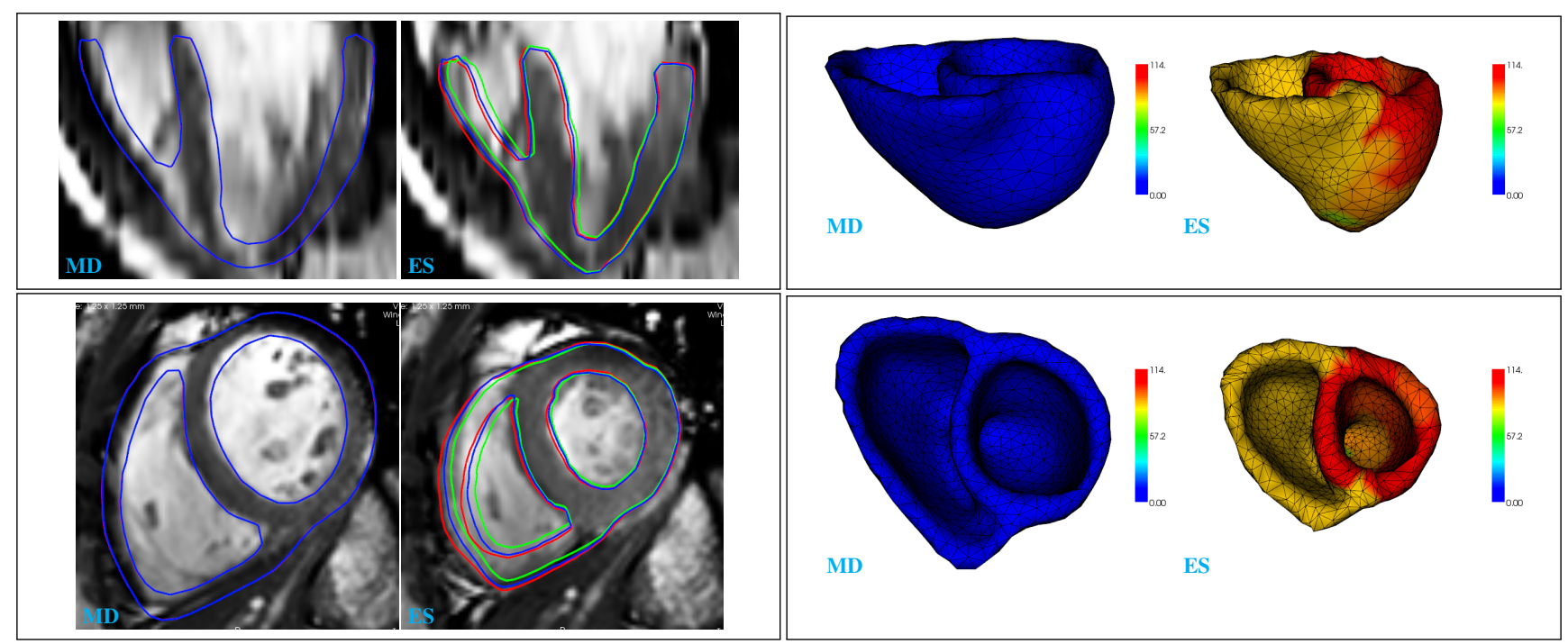

(a) Volunteer 1.

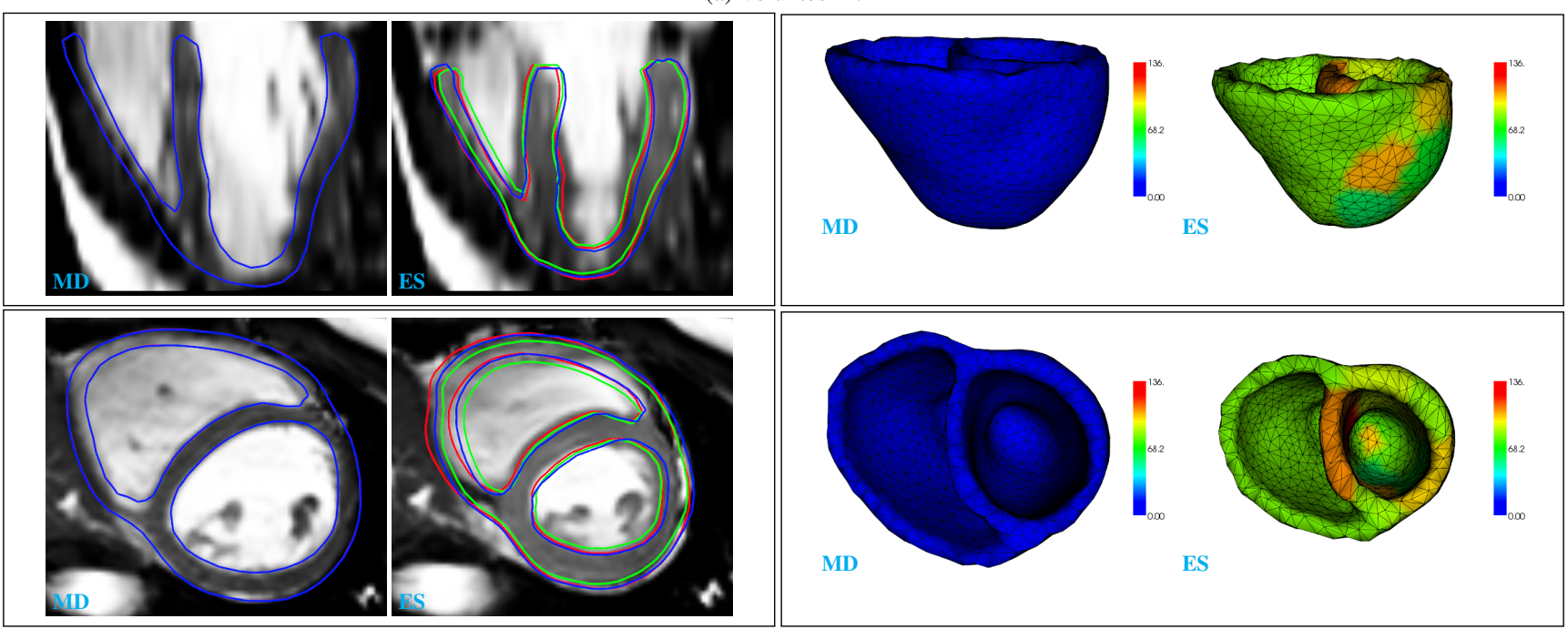

(b) Volunteer 2.

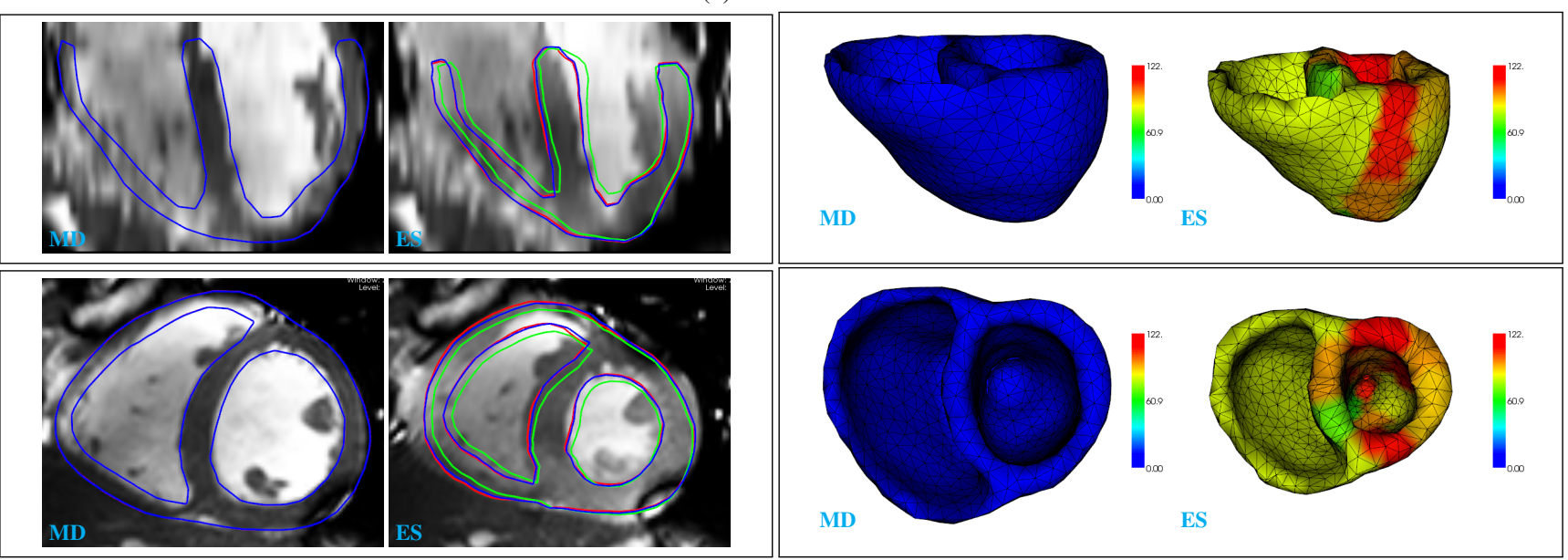

(c) Volunteer 3.

Figure 11: Volunteer data. Results at mid diastole (MD) and end systole (ES). Left: heart geometries overlapped with images, with red, green, and blue representing personalized kinematics, initializations, and personalized simulations respectively. Right: personalized simulations and the corresponding contraction stresses in $\mathrm{kPa}$. 

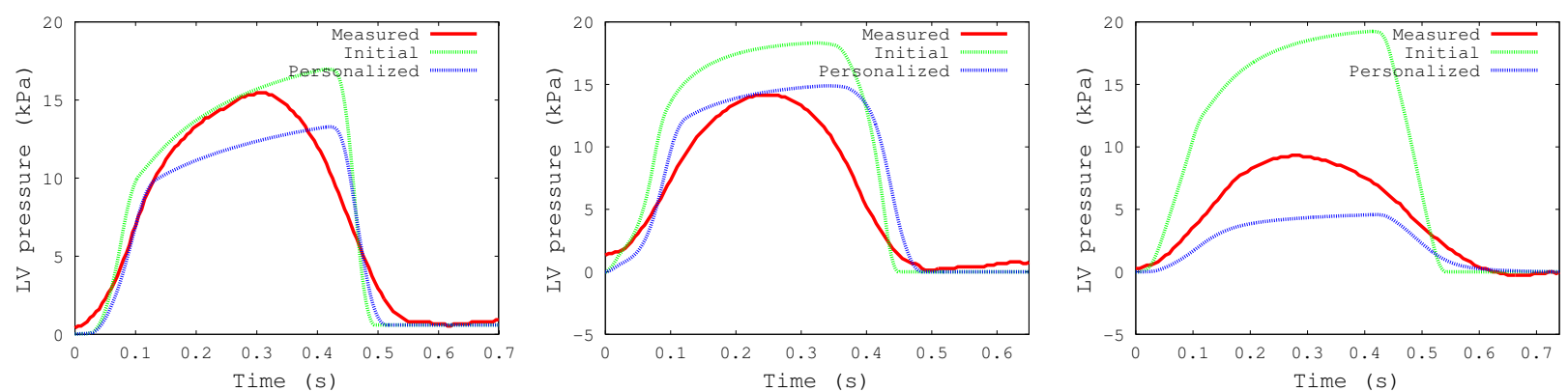

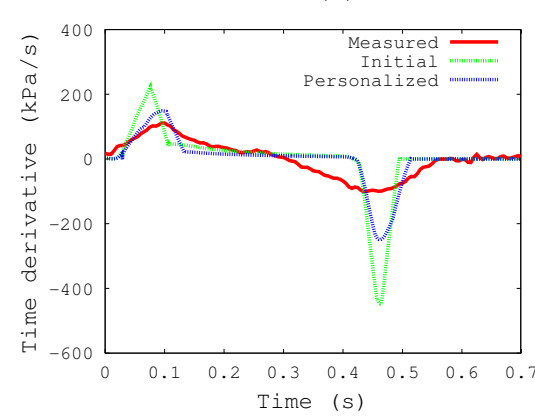

(a) Patient 1 .

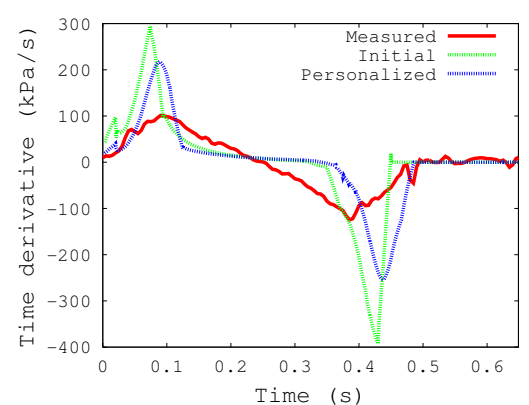

(b) Patient 2

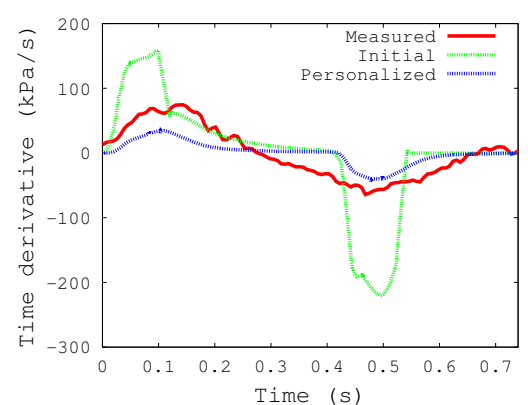

(c) Patient 3

Figure 12: Patient data. Results of velocity-based optimization. Top: LV pressures. Bottom: the corresponding time derivatives.

model parameters were used for all patients in the experiments for fair comparison. The time derivatives of the blood pressures are also compared because they are important indicators of cardiac contractility (Rushmer et al., 1964; Mason et al., 1971). The LV blood pressures and their time derivatives show large improvement after mechanics personalization, especially for Patient 2 and 3. Because of the limitations of our current model, such as the accurate determination of the valve opening and closing, the shapes of the simulated pressure curves do not perfectly match with those of the measurements during the ejection phase. Nevertheless, the improvement can still show that the personalized electromechanical models can partially reflect the subjects' actual physiologies.

Table 5 and 6 show the estimated parameters, Figure 13 and 14 show the corresponding bullseye plots of the estimated AHA zonal parameters, and Figure 15 provides their box plots. For Patient 1 (5-zone representation), consistent to Figure 10, the maximum contraction parameters $\left(\sigma_{0}\right)$ are larger at the LV and RV, but smaller at the scar, septum, and apex. For the patient data, the variations among zones and patients are relatively large. For example, the average $\sigma_{0}$ range from 33.0 to $85.2 \mathrm{kPa}$, with the largest standard deviation as $40.1 \mathrm{kPa}$. On the other hand, the variations of the volunteer data are much lower, with average $\sigma_{0}$ range from 92.1 to $97.7 \mathrm{kPa}$, with the largest standard deviation as 18.5. Similar patterns can also be observed for the contraction and relaxation rates, and the differences between the populations are clearly shown in the box plots in Figure 15 .

From Table 5, there are three estimated parameters of Patient 3 equal zeros (zone 3, 8, and 9). For zone 8 and 9, the zero values imply no contraction. For zone 3 , as the contraction rate is nearly zero, the zero relaxation rate has no contribution. As patient 3 has severe dilated cardiomyopathy, these zero val- ues are reasonable. On the other hand, from Table 6, Volunteer 1 has zero relaxation rate at zone 8 , and Volunteer 2 and 3 also have small relaxation rates at zone 8 . The actual reason of this is unclear, but zone 8 corresponds to the mid-anteroseptal region, which has relatively complicated geometry connecting the left and right ventricles. Therefore, realistic simulation can be relatively difficult at zone 8 and this may lead to suboptimal estimation.

\section{Discussion}

The experimental results on the synthetic data show that the use of velocity-based optimization outperforms the positionbased one. Nevertheless, on clinical data, it is difficult to justify which objective functions or algorithms provide better results. We may justify the realism of a model through its ability of prediction, for example, one can simulate the cardiac resynchronization therapy procedure on a personalized model to see if the prediction matches the interventional measurements (Sermesant et al., 2012). Nevertheless, such a validation is very complicated as it requires the personalization of electrophysiology, active contraction, and passive mechanical properties, and also a realistic simulation of the therapy procedure. Therefore, in this paper, our main validation is based on the synthetic experiments to assess the best possible performance of the framework in ideal situations.

Although Figure 12 shows relative improvements of the simulated pressures using the personalized models, without incorporating measurements which can aid the personalization of the pressure-related parameters such as those of the Windkessel model, accurate prediction of the absolute pressure values may be infeasible. Although blood pressure measurements are available in the diseased cases which may be incorporated to identify 
Table 5: Patient data. Estimated contraction parameters.

\begin{tabular}{|c|c|c|c|c|c|c|}
\hline \multicolumn{7}{|c|}{ Patient 1 (velocity-based) } \\
\hline Zone & LV & RV & Scar & Septum & Apex & Average \\
\hline$\sigma_{0}(\mathrm{kPa})$ & 80.7 & 80.0 & 48.0 & 52.2 & 43.7 & $60.9 \pm 18.0$ \\
\hline$\alpha_{c}\left(\mathrm{~s}^{-1}\right)$ & 29.5 & 13.6 & 34.7 & 45.4 & 32.3 & $31.1 \pm 11.5$ \\
\hline$\alpha_{r}\left(\mathrm{~s}^{-1}\right)$ & 34.0 & 29.4 & 49.8 & 6.3 & 50.1 & $33.9 \pm 18.0$ \\
\hline
\end{tabular}

\begin{tabular}{|c|c|c|c|c|c|c|c|c|c|c|c|c|c|c|c|c|c|c|c|}
\hline \multicolumn{10}{|c|}{ Patient 2 (velocity-based) } & \multicolumn{10}{|c|}{10} \\
\hline Zone & 1 & 2 & 3 & 4 & 5 & 6 & 7 & 8 & 9 & 10 & 11 & 12 & 13 & 14 & 15 & 16 & 17 & RV & Average \\
\hline$\sigma_{0}(\mathrm{kPa})$ & 61.6 & 95.2 & 39.6 & 78.3 & 71.8 & 86.5 & 131.9 & 166.3 & 172.4 & 79.2 & 64.1 & 68.0 & 43.9 & 71.4 & 56.6 & 48.6 & 118.2 & 79.4 & $85.2 \pm 38.6$ \\
\hline$\alpha_{c}\left(\mathrm{~s}^{-1}\right)$ & 9.8 & 6.0 & 32.3 & 25.0 & 34.0 & 17.2 & 8.7 & 11.5 & 11.5 & 29.8 & 31.3 & 31.6 & 20.2 & 15.7 & 19.7 & 26.1 & 16.2 & 8.0 & $19.7 \pm 9.5$ \\
\hline$\alpha_{r}\left(\mathrm{~s}^{-1}\right)$ & 10.4 & 13.2 & 28.5 & 24.6 & 26.9 & 26.4 & 13.8 & 17.4 & 19.1 & 21.5 & 56.2 & 34.7 & 16.2 & 15.9 & 17.8 & 36.0 & 17.8 & 9.8 & $22.6 \pm 11.3$ \\
\hline
\end{tabular}

\begin{tabular}{|c|c|c|c|c|c|c|c|c|c|c|c|c|c|c|c|c|c|c|c|}
\hline \multicolumn{10}{|c|}{ Patient 3 (velocity-based) } \\
\hline Zone & 1 & 2 & 3 & 4 & 5 & 6 & 7 & 8 & 9 & 10 & 11 & 12 & 13 & 14 & 15 & 16 & 17 & RV & Average \\
\hline$\sigma_{0}(\mathrm{kPa})$ & 19.3 & 12.2 & 10.4 & 24.2 & 30.8 & 32.7 & 48.9 & 183.1 & 0.0 & 55.8 & 14.2 & 21.1 & 14.1 & 36.1 & 11.8 & 17.0 & 24.3 & 37.9 & $33.0 \pm 40.1$ \\
\hline$\alpha_{c}\left(\mathrm{~s}^{-1}\right)$ & 26.4 & 9.9 & 0.9 & 3.7 & 16.0 & 27.3 & 5.8 & 0.0 & 49.4 & 2.3 & 70.2 & 38.0 & 24.9 & 8.1 & 42.1 & 47.5 & 30.7 & 9.8 & $22.9 \pm 20.2$ \\
\hline$\alpha_{r}\left(\mathrm{~s}^{-1}\right)$ & 47.5 & 3.7 & 0.0 & 56.7 & 37.8 & 28.4 & 44.8 & 181.3 & 26.9 & 8.2 & 46.2 & 42.6 & 2.5 & 19.0 & 47.1 & 86.0 & 30.4 & 18.0 & $40.4 \pm 41.4$ \\
\hline
\end{tabular}

Table 6: Volunteer data. Estimated contraction parameters.

\begin{tabular}{|c|c|c|c|c|c|c|c|c|c|c|c|c|c|c|c|c|c|c|c|}
\hline \multicolumn{20}{|c|}{ Volunteer 1 (velocity-based) } \\
\hline Zone & 1 & 2 & 3 & 4 & 5 & 6 & 7 & 8 & 9 & 10 & 11 & 12 & 13 & 14 & 15 & 16 & 17 & RV & Average \\
\hline$\overline{\sigma_{0}(\mathrm{kPa})}$ & 108.0 & 110.2 & 105.2 & 102.4 & 96.4 & 109.6 & 89.0 & 117.7 & 110. & 96. & 97.7 & 100. & 89. & $\overline{73.0}$ & 88. & 89.1 & 90.3 & 84.8 & $97.7 \pm 11.3$ \\
\hline$\alpha_{c}\left(\mathrm{~s}^{-1}\right)$ & 56.2 & 22.7 & 28.1 & 28.1 & 33.6 & 26.2 & 58.2 & 11.8 & 13.6 & 37. & 34.2 & 32.1 & 31. & $\overline{40.6}$ & 28. & \begin{tabular}{l|l}
4 & 28.3 \\
\end{tabular} & 26.8 & 28.9 & $31.5 \pm 11.7$ \\
\hline$\alpha_{r}\left(\mathrm{~s}^{-1}\right)$ & 27.9 & 31.0 & 27.5 & 28.8 & 31.7 & 25.1 & 41.9 & 0.0 & 18.1 & 24. & 28.6 & 33.8 & 39. & 14.3 & 33. & \begin{tabular}{l|l|l}
.6 & 38.0 \\
\end{tabular} & 0.5 & 32.3 & $26.5 \pm 11.7$ \\
\hline \multicolumn{20}{|c|}{ Volunteer 2 (velocity-based) } \\
\hline Zone & 1 & 2 & 3 & 4 & 5 & 6 & 7 & 8 & 9 & 10 & 11 & 12 & 13 & 14 & 15 & 16 & 17 & RV & Average \\
\hline$\sigma_{0}(\mathrm{kPa})$ & 85.7 & 108.0 & 108.6 & 91.2 & 84.3 & 99.6 & 104.4 & 112.8 & 136.8 & 90.3 & 90.6 & 65.1 & 57.8 & 81.5 & 77.8 & 80.6 & 101.2 & 81.7 & $92.1 \pm 18.5$ \\
\hline$\alpha_{c}\left(\mathrm{~s}^{-1}\right)$ & 62.6 & 32.1 & 22.6 & 29.4 & 41.4 & 48.9 & 43.1 & 27.2 & 20.4 & 26.1 & 42.0 & 51.1 & 69.7 & 19.7 & 20.8 & 45.8 & 27.9 & 25.0 & $36.4 \pm 14.9$ \\
\hline$\alpha_{r}\left(\mathrm{~s}^{-1}\right)$ & 42.1 & 42.8 & 35.9 & 32.2 & 36.9 & 43.3 & 29.4 & 3.2 & 20.9 & 19.1 & 40.4 & 46.5 & 19.3 & 8.4 & 40.1 & 26.6 & 5.3 & 28.8 & $29.0 \pm 13.6$ \\
\hline \multicolumn{20}{|c|}{ Volunteer 3 (velocity-based) } \\
\hline Zone & 1 & 2 & 3 & 4 & 5 & 6 & 7 & \begin{tabular}{|l|}
8 \\
\end{tabular} & 9 & 10 & 11 & 12 & 13 & 14 & 15 & 16 & 17 & $\mathrm{RV}$ & Average \\
\hline$\sigma_{0}(\mathrm{kPa})$ & 108.7 & 70.9 & 93.9 & 110.1 & 94.6 & 89.6 & 107.8 & 65.1 & 128.2 & 95.4 & 114.6 & 83.9 & 95.2 & 64.6 & 98.3 & 83.6 & 107.4 & 81.9 & $94.1 \pm 17.3$ \\
\hline$\alpha_{c}\left(\mathrm{~s}^{-1}\right)$ & 38.5 & 46.9 & 29.1 & 30.1 & 32.6 & 29.7 & 46.0 & 33.7 & 10.0 & 40.7 & 25.0 & 42.2 & 22.0 & 39.3 & 21.3 & 29.4 & 20.1 & 25.9 & $31.3 \pm 9.8$ \\
\hline$\alpha_{r}\left(\mathrm{~s}^{-1}\right)$ & 33.5 & 53.2 & 36.0 & 28.3 & 32.6 & 35.6 & 30.2 & 3.2 & 2.6 & 27.0 & 36.9 & 43.5 & 30.3 & 58.4 & 29.3 & 28.4 & 25.4 & 33.0 & $31.5 \pm 13.6$ \\
\hline
\end{tabular}

such parameters, we have not utilized them in the personalization. One reason is that such invasive measurements may not be always available, such as the volunteers' data. Another reason is that it is nontrivial to incorporate pressure data. If we directly apply the pressure measurements as the natural boundary conditions on the endocardial surfaces, the simulations will be more unstable as the heart geometry may collapse because of the inconsistency between the active contractility and the applied pressure. A more flexible way is to embed the pressure data into the objective function, which will be investigated in the future.

This framework is similar to other parameter estimation frameworks (Chabiniok et al., 2011; Delingette et al., 2012; Liu and Shi, 2009; Wang et al., 2009; Xi et al., 2011), and therefore suffers from the curse of dimensionality. Although the subplex method has already helped to alleviate the problem by decomposing the high-dimensional space into subspaces, the numbers of iterations required are still large, and it will be computationally very challenging with more number of zones. To address this problem, one possibility is to study the spatial dependencies among different regions to see if hierarchical approaches can be applied. Furthermore, the interactions between parameters within a zone can also be investigated for the possibility of decomposing the estimation, so that the search space can be reduced.

Some more limitations of this framework. First of all, the passive mechanical properties are assumed to be known and homogeneous, but the myocardium is known to be heterogeneous especially in diseased cases. As the reduction of myocardial deformation can be caused by hardening of material, reduction of contractility, or both (Braunwald et al., 2001; Germann and Stanfield, 2005; Glass et al., 1991), the assumption of known material properties can lead to suboptimal parameter estimation. In fact, the framework of passive mechanical parameter estimation is also in progress (Wong et al., 2012), which can be combined with the framework in this paper when both become mature, though the task will be very challenging. Secondly, some of the calibrations were performed by manual adjustments, for example, the time differences between the electrical excitation and the onset of the active contraction. Techniques such as that in Marchesseau et al. (2013) can be used to alleviate this issue. Thirdly, we use the cine MRI with the electromechanical model to provide the velocity measurements because image modalities such as phase-contrast MRI are unavailable in our data sets. If such image modalities are available to directly provide the velocity measurements, more patientspecific results can be expected as the model uncertainties are reduced. 


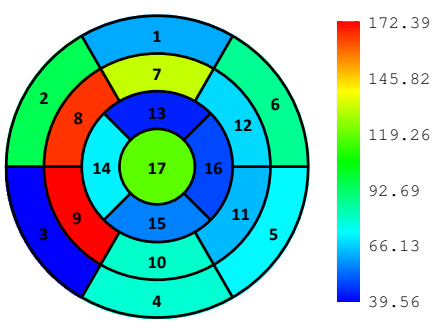

$\sigma_{0}(\mathrm{kPa})$

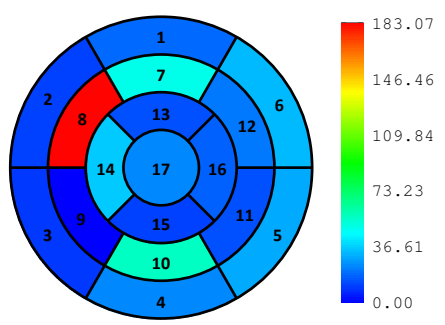

$\sigma_{0}(\mathrm{kPa})$

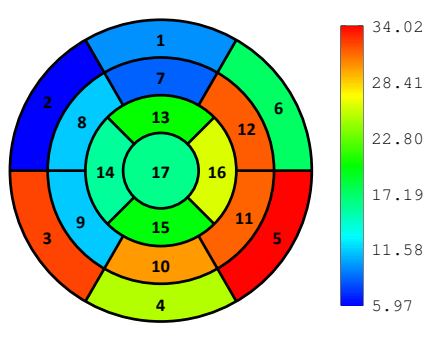

$\alpha_{c}\left(\mathrm{~s}^{-1}\right)$

(a) Patient 2

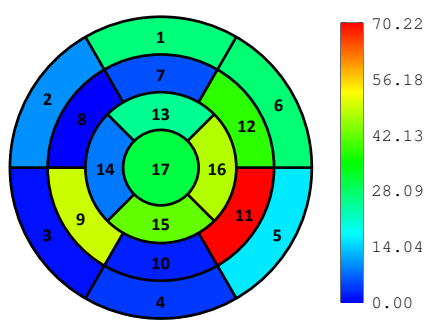

$\alpha_{c}\left(\mathrm{~s}^{-1}\right)$

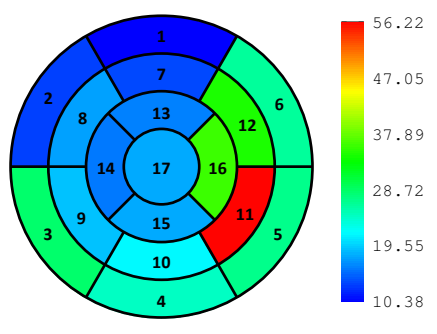

$\alpha_{r}\left(\mathrm{~s}^{-1}\right)$

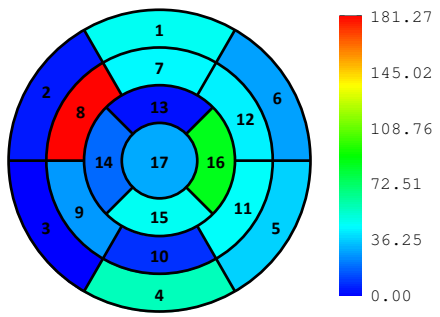

$\alpha_{r}\left(\mathrm{~s}^{-1}\right)$

(b) Patient 3

Figure 13: Patient data. Bullseye plots corresponding to the estimated AHA zonal parameters in Table 5.

\section{Conclusion}

We presented a velocity-based cardiac contractility personalization framework using the derivative-free subplex optimization. With derivative-free optimization, analytical and numerical difficulties associated with gradient evaluation can be avoided, and an objective function accounting for the velocity differences between simulations and measurements was proposed to personalize cardiac contractility. Experiments on synthetic data show that the velocity-based optimization outperforms the positionbased one for simultaneous estimation of the maximum contraction parameters, contraction rates, and relaxation rates. Experiments on clinical data show that the proposed framework can provide personalized simulations which are consistent with the subject-specific physiologies of both patients and healthy volunteers.

\section{Acknowledgements}

Part of this work was funded by the advanced ERC grant MedYMA.

\section{References}

Bathe, K. J., 1996. Finite Element Procedures. Prentice Hall.

Belytschko, T., Krongauz, Y., Organ, D., Fleming, M., Krysl, P., 1996. Meshless methods: an overview and recent developments. Computer Methods in Applied Mechanics and Engineering 139 (1-4), 3-47.

Bestel, J., Clément, F., Sorine, M., 2001. A biomechanical model of muscle contraction. In: International Conference on Medical Image Computing and Computer Assisted Intervention. Vol. 2208 of LNCS. Springer, pp. 11591161.

Billet, F., Sermesant, M., Delingette, H., Ayache, N., 2009. Cardiac motion recovery and boundary conditions estimation by coupling an electromechanical model and cine-MRI data. In: International Conference on Functional Imaging and Modeling of the Heart. Vol. 5528 of LNCS. Springer, pp. 376385 .
Braunwald, E., Zipes, D. P., Libby, P. (Eds.), 2001. Heart Disease: A Textbook of Cardiovascular Medicine, 6th Edition. W.B. Saunders Company.

Cerqueira, M. D., Weissman, N. J., Dilsizian, V., Jacobs, A. K., Kaul, S., Laskey, W. K., Pennell, D. J., Rumberger, J. A., Ryan, T., Verani, M. S., 2002. Standardized myocardial segmentation and nomenclature for tomographic imaging of the heart: a statement for healthcare professionals from the cardiac imaging committee of the council on clinical cardiology of the American Heart Association. Circulation 105, 539-542.

Chabiniok, R., Moireau, P., Lesault, P.-F., Rahmouni, A., Deux, J.-F., Chapelle, D., 2011. Trials on tissue contractility estimation from cardiac cine MRI using a biomechanical heart model. In: International Conference on Functional Imaging and Modeling of the Heart. Vol. 6666 of LNCS. Springer, pp. 304-312.

Conn, A. R., Scheinberg, K., Vicente, L. N., 2009. Introduction to DerivativeFree Optimization. Vol. 8. Society for Industrial Mathematics.

Delingette, H., Billet, F., Wong, K. C. L., Sermesant, M., Rhode, K., Ginks, M., Rinaldi, C. A., Razavi, R., Ayache, N., 2012. Personalization of cardiac motion and contractility from images using variational data assimilation. IEEE Transactions on Biomedical Engineering 59 (1), 20-24.

Germann, W. J., Stanfield, C. L., 2005. Principles of Human Physiology. Pearson Benjamin Cummings.

Glass, L., Hunter, P., McCulloch, A. (Eds.), 1991. Theory of Heart: Biomechanics, Biophysics, and Nonlinear Dynamics of Cardiac Function. Springer-Verlag.

Hu, Z., Metaxas, D., Axel, L., 2003. In vivo strain and stress estimation of the heart left and right ventricles from MRI images. Medical Image Analysis 7 (4), 435-444.

Liu, H., Shi, P., 2009. Maximum a posteriori strategy for the simultaneous motion and material property estimation of the heart. IEEE Transactions on Biomedical Engineering 56 (2), 378-389.

Marchesseau, S., Delingette, H., Sermesant, M., Cabrera-Lozoya, R., TobonGomez, C., Moireau, P., Figueras i Ventura, R. M., Lekadir, K., Hernandez, A., Garreau, M., Donal, E., Leclercq, C., Duckett, S. G., Rhode, K., Rinaldi, C., Frangi, A. F., Razavi, R., Chapelle, D., Ayache, N., 2013. Personalization of a cardiac electromechanical model using reduced order unscented Kalman filtering from regional volumes. Medical Image Analysis 17 (7), 816-829.

Mason, D. T., Braunwald, E., Covell, J. W., Sonnenblick, E. H., Ross, J., 1971. Assessment of cardiac contractility: the relation between the rate of pressure rise and ventricular pressure during isovolumic systole. Circulation 44 (1), 47-58.

Mitchell, C. C., Schaeffer, D. G., 2003. A two-current model for the dynamics 


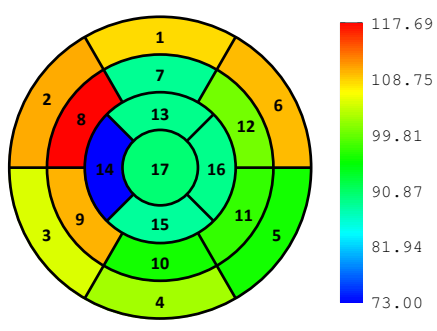

$\sigma_{0}(\mathrm{kPa})$

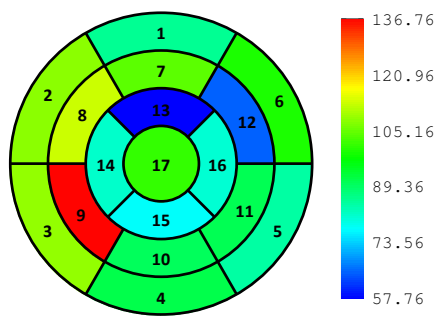

$\sigma_{0}(\mathrm{kPa})$

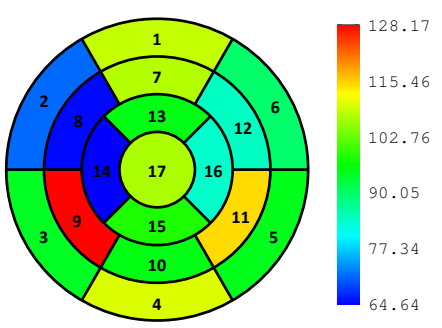

$\sigma_{0}(\mathrm{kPa})$

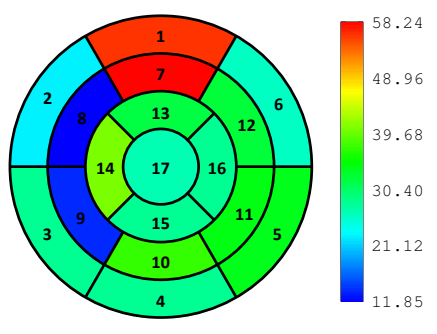

$\alpha_{c}\left(\mathrm{~s}^{-1}\right)$

(a) Volunteer 1.

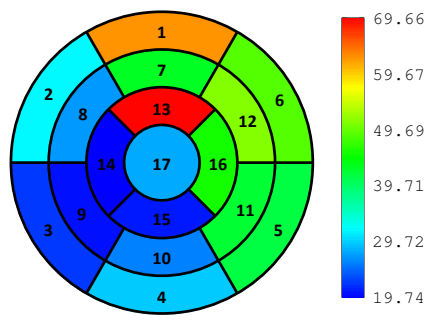

$\alpha_{c}\left(\mathrm{~s}^{-1}\right)$

(b) Volunteer 2.

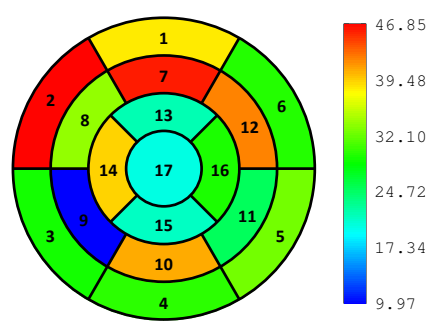

$\alpha_{c}\left(\mathrm{~s}^{-1}\right)$

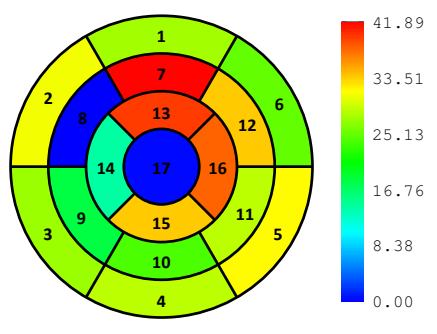

$\alpha_{r}\left(\mathrm{~s}^{-1}\right)$

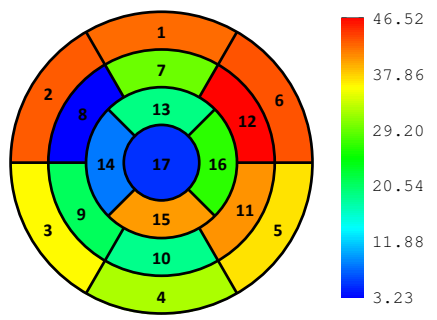

$\alpha_{r}\left(\mathrm{~s}^{-1}\right)$

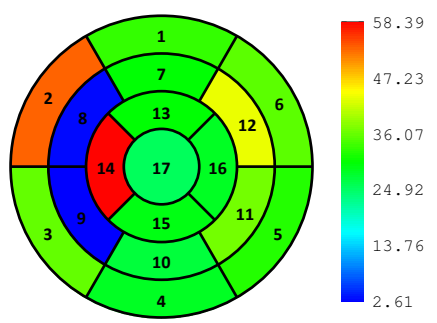

$\alpha_{r}\left(\mathrm{~s}^{-1}\right)$

(c) Volunteer 3

Figure 14: Volunteer data. Bullseye plots corresponding to the estimated AHA zonal parameters in Table 6.
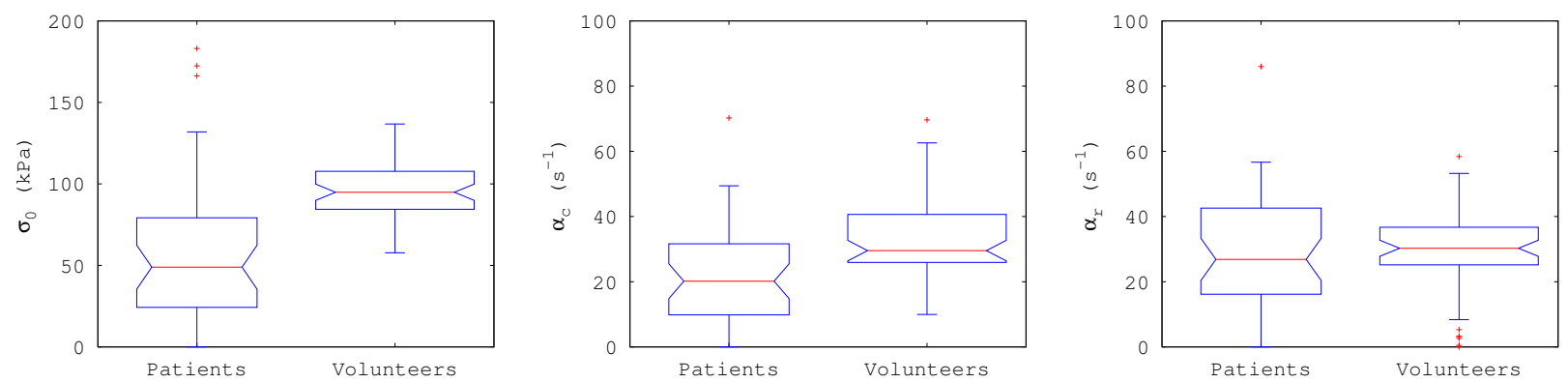

Figure 15: Clinical data. Box plots of the estimated parameters of all patients and volunteers. Left to right: maximum contraction parameter $\sigma_{0}$, contraction rate $\alpha_{c}$, and relaxation rate $\alpha_{r}$.

of cardiac membrane. Bulletin of Mathematical Biology 65 (5), 767-793.

Moireau, P., Chapelle, D., Le Tallec, P., 2008. Joint state and parameter estimation for distributed mechanical systems. Computer Methods in Applied Mechanics and Engineering 197 (6-8), 659-677.

Nash, M., 1998. Mechanics and material properties of the heart using an anatomically accurate mathematical model. Ph.D. thesis, The University of Auckland.

Nelder, J. A., Mead, R., 1965. A simplex method for function minimization. Computer Journal 7 (4), 308-313.

Powell, M. J. D., 2003. On trust region methods for unconstrained minimization without derivatives. Mathematical Programming 97 (3), 605-623.

Rao, S. S., 2009. Engineering Optimization: Theory and Practice. John Wiley
\& Sons, Inc.

Relan, J., Chinchapatnam, P., Sermesant, M., Rhode, K., Ginks, M., Delingette, H., Rinaldi, C. A., Razavi, R., Ayache, N., 2011. Coupled personalization of cardiac electrophysiology models for prediction of ischaemic ventricular tachycardia. Journal of the Royal Society Interface Focus 1 (3), 396-407.

Rhode, K., Sermesant, M., Brogan, D., Hegde, S., Hipwell, J., Lambiase, P., Rosenthal, E., Bucknall, C., Qureshi, S., Gill, J., Razavi, R., Hill, D., 2005. A system for real-time XMR guided cardiovascular intervention. IEEE Transactions on Medical Imaging 24 (11), 1428-1440.

Rowan, T., 1990. Functional stability analysis of numerical algorithms. Ph.D. thesis, University of Texas at Austin.

Rushmer, R. F., Harding, D., Baker, D., Watson, N., 1964. Initial ventricular 
impulse: a potential key to cardiac evaluation. Circulation 29 (2), 268-283.

Sermesant, M., Billet, F., Chabiniok, R., Mansi, T., Chinchapatnam, P., Moireau, P., Peyrat, J., Rhode, K., Ginks, M., Lambiase, P., Arridge, S., Delingette, H., Sorine, M., Rinaldi, C., Chapelle, D., Razavi, R., Ayache, N., 2009. Personalised electromechanical model of the heart for the prediction of the acute effects of cardiac resynchronisation therapy. In: International Conference on Functional Imaging and Modeling of the Heart. Vol. 5528 of LNCS. Springer, pp. 239-248.

Sermesant, M., Chabiniok, R., Chinchapatnam, P., Mansi, T., Billet, F., Moireau, P., Peyrat, J.-M., Wong, K. C. L., Relan, J., Rhode, K. S., Ginks, M., Lambiase, P., Delingette, H., Sorine, M., Rinaldi, C., Chapelle, D., Razavi, R., Ayache, N., 2012. Patient-specific electromechanical models of the heart for the prediction of pacing acute effects in CRT: A preliminary clinical validation. Medical Image Analysis 16 (1), 201-215.

Sermesant, M., Delingette, H., Ayache, N., 2006a. An electromechanical model of the heart for image analysis and simulation. IEEE Transactions on Medical Imaging 25 (5), 612-625.

Sermesant, M., Moireau, P., Camara, O., Sainte-Marie, J., Andriantsimiavona, R., Cimrman, R., Hill, D. L. G., Chapelle, D., Razavi, R., 2006b. Cardiac function estimation from MRI using a heart model and data assimilation: advances and difficulties. Medical Image Analysis 10, 642-656.

Sundar, H., Davatzikos, C., Biros, G., 2009. Biomechanically-constrained 4D estimation of myocardial motion. In: International Conference on Medical Image Computing and Computer Assisted Intervention. Vol. 5762 of LNCS. Springer, pp. 257-265.

Usyk, T. P., LeGrice, I. J., McCulloch, A. D., 2002. Computational model of three-dimensional cardiac electromechanics. Computing and Visualization in Science 4 (4), 249-257.

Wang, V. Y., Lam, H. I., Ennis, D. B., Cowan, B. R., Young, A. A., Nash, M. P., 2009. Modelling passive diastolic mechanics with quantitative MRI of cardiac structure and function. Medical Image Analysis 13 (5), 773-784.

Wong, K. C. L., Billet, F., Mansi, T., Chabiniok, R., Sermesant, M., Delingette, H., Ayache, N., 2010a. Cardiac motion estimation using a proactive deformable model: evaluation and sensitivity analysis. In: Statistical Atlases and Computational Models of the Heart. Vol. 6364 of LNCS. Springer, pp. 154-163.

Wong, K. C. L., Relan, J., Wang, L., Sermesant, M., Delingette, H., Ayache, N., Shi, P., 2012. Strain-based regional nonlinear cardiac material properties estimation from medical images. In: International Conference on Medical Image Computing and Computer Assisted Intervention. Vol. 7510 of LNCS. Springer, pp. 617-624.

Wong, K. C. L., Wang, L., Zhang, H., Liu, H., Shi, P., 2010b. Meshfree implementation of individualized active cardiac dynamics. Computerized Medical Imaging and Graphics 34 (1), 91-103.

Xi, J., Lamata, P., Lee, J., Moireau, P., Chapelle, D., Smith, N., 2011. Myocardial transversely isotropic material parameter estimation from in-silico measurements based on reduced-order unscented Kalman filter. Journal of the Mechanical Behavior of Biomedical Materials 4 (7), 1090-1102. 